Алгебра и анализ

Toм 19 (2007), № 6
St. Petersburg Math. J. Vol. 19 (2008), No. 6, Pages 883-910 S 1061-0022(08)01026-1

Article electronically published on August 21, 2008

\title{
ON (2,3)-GENERATION OF MATRIX GROUPS OVER THE RING OF INTEGERS
}

\author{
M. A. VSEMIRNOV
}

To the centenary of D. K. Faddeev's birth

\begin{abstract}
The groups $\mathrm{GL}(5, \mathbb{Z}), \mathrm{SL}(5, \mathbb{Z}), \mathrm{SL}(6, \mathbb{Z}), \mathrm{GL}(7, \mathbb{Z})$ and $\mathrm{SL}(7, \mathbb{Z})$ are $(2,3)$ generated.
\end{abstract}

\section{$\S 1$. INTRODUCTION}

A group is said to be $(2,3)$-generated if it can be generated by an involution and an element of order 3 . It is well known that the group $\operatorname{PSL}(2, \mathbb{Z})$ is isomorphic to the free product of the cyclic group of order 2 and the cyclic group of order $3: \operatorname{PSL}(2, \mathbb{Z}) \simeq C_{2} * C_{3}$. Therefore, the problem of classification of $(2,3)$-generated groups is, in essence, the problem of finding the quotients of the modular group different from $\{1\}, C_{2}$, and $C_{3}$. In this generality, the problem looks absolutely hopeless. Thus, what is usually studied is $(2,3)$-generation in some wide class of groups.

Many authors investigated the problem of $(2,3)$-generation for classical matrix groups over finite fields $[1,2,4,9,10$ and over the ring $\mathbb{Z}$ of integers $[5,7,18,11,12,13$. In the case of groups over finite fields the problem is solved (see, e.g., 4]). However, for matrix groups over $\mathbb{Z}$, the answer is not known for all dimensions.

As was shown in $[8$, the groups $\operatorname{SL}(n, \mathbb{Z})$ and $\operatorname{GL}(n, \mathbb{Z})$ are $(2,3)$-generated provided $n \geq 13$ and $n \geq 19$, respectively. On the other hand, $\mathrm{SL}(4, \mathbb{Z})$ and $\mathrm{GL}(4, \mathbb{Z})$ are not $(2,3)$ generated, since even the group $\mathrm{SL}(4,2) \simeq \mathrm{GL}(4,2) \simeq \operatorname{Alt}(8)$ is not $(2,3)$-generated [6]. It is also known that $\mathrm{SL}(3, \mathbb{Z})$ and $\mathrm{GL}(3, \mathbb{Z})$ are not $(2,3)$-generated $[\mathbf{7}, 11$.

For the groups $\mathrm{GL}(5, \mathbb{Z})$ and $\mathrm{SL}(6, \mathbb{Z})$, some preliminary results were given in $[5]$ and [12, respectively. Those papers did not give a complete answer, but described a reduction of the original problem to the analysis of finitely many cases listed explicitly.

For a long time it has not even been clear where the border between the positive and negative results lies. In a private correspondence, M. C. Tamburini conjectured that the groups $\mathrm{SL}(n, \mathbb{Z})$ for $n \leq 12$ might be non-(2,3)-generated. In this respect, the author's result in [13], showing that the group $\mathrm{GL}(6, \mathbb{Z})$ can be generated by an involution and an element of order 3 , was all the more astonishing.

In this paper we present further positive results on $(2,3)$-generation of the groups $\mathrm{SL}(n, \mathbb{Z})$ and $\mathrm{GL}(n, \mathbb{Z})$. In particular we prove the following theorem.

Theorem 1.1. The groups $\mathrm{GL}(5, \mathbb{Z}), \mathrm{SL}(5, \mathbb{Z}), \mathrm{SL}(6, \mathbb{Z}), \mathrm{GL}(7, \mathbb{Z})$, and $\mathrm{SL}(7, \mathbb{Z})$ are $(2,3)$-generated.

2000 Mathematics Subject Classification. Primary 20G30; Secondary 20F05, 20C12.

Key words and phrases. (2,3)-generation, linear groups.

Supported by the RAS program of fundamental research "Modern problems of theoretical mathematics" and by the "Scientific schools" program (grant no. NSh-8464-2006-1). 
This result is proved separately for each value of $n$; see Theorem 1.2 for $n=5$, Theorem 7.1 for $n=6$ and Theorem 9.1 for $n=7$ below. In addition, in Theorem 8.1 we construct yet another set of $(2,3)$-generators for the group $\operatorname{PSL}(6, \mathbb{Z})$, which is not inherited from $\mathrm{SL}(6, \mathbb{Z})$.

For $n=5$ we can achieve more. Namely, for $\mathrm{GL}(5, \mathbb{Z})$ and $\mathrm{SL}(5, \mathbb{Z})$ we list all pairs of $(2,3)$-generators up to $\mathrm{GL}(5, \mathbb{Z})$-conjugation.

Theorem 1.2. The groups $\mathrm{GL}(5, \mathbb{Z})$ and $\mathrm{SL}(5, \mathbb{Z})$ are $(2,3)$-generated. Moreover, any pair of $(2,3)$-generators of $\mathrm{GL}(5, \mathbb{Z})$ is conjugate in $\mathrm{GL}(5, \mathbb{Z})$ to one of the six pairs of matrices $X, Y$, while any pair of $(2,3)$-generators of $\mathrm{SL}(5, \mathbb{Z})$ is conjugate in $\mathrm{GL}(5, \mathbb{Z})$ to one of the six pairs of matrices $-X, Y$, where

$$
X=\left(\begin{array}{rrrrr}
-1 & 0 & 0 & 0 & 0 \\
0 & -1 & 0 & 0 & 0 \\
0 & 0 & -1 & 0 & 0 \\
1 & 0 & 0 & 1 & 0 \\
0 & 0 & 1 & 0 & 1
\end{array}\right), \quad Y=\left(\begin{array}{rrrrr}
0 & 1 & 0 & 0 & a_{1} \\
-1 & -1 & 0 & 0 & a_{2} \\
0 & 0 & 0 & 1 & a_{3} \\
0 & 0 & -1 & -1 & a_{4} \\
0 & 0 & 0 & 0 & 1
\end{array}\right)
$$

and $\left(a_{1}, a_{2}, a_{3}, a_{4}\right)$ is one of the following sets:

$$
\begin{array}{cl}
(1,-1,-2,-2), & (0,-1,-2,-2), \\
(-1,1,-2,-2), & (0,1,-2,-2), \\
(1,-1,1,-3), & (0,-1,0,-1) .
\end{array}
$$

The proof of Theorem 1.2 is given in $\S ₫ 36$, in particular, see Theorems 3.1, 3.2. 3.3. 4.1, 5.1, and 6.1.

The results of the present paper, together with the results of [8, give hope for the complete solution of the (2,3)-generation problem for the groups $\operatorname{SL}(n, \mathbb{Z})$ and $\operatorname{GL}(n, \mathbb{Z})$. For the group $\operatorname{SL}(6, \mathbb{Z})$, a classification similar to Theorem 1.2 may be expected. For partial results in this direction, see 12 . However, at the moment I am unable to give a complete answer.

Some matrix manipulations used in the proofs of the main results of the present paper were made by means of the MAGMA package.

The author is grateful to N. A. Vavilov and M. C. Tamburini for useful discussions.

\section{§2. Notation AND AUXiliary STAtements}

In the present paper we use the following notation. The identity matrix of size $n$ is denoted by $I_{n}$ or simply by $I$ if the size is clear from the context. As usual, the $e_{i j}$, $1 \leq i, j \leq n$, are the elements of the standard basis of the matrix algebra (the so-called "matrix units") and the $t_{i j}(\xi)$ are the elementary transvections

$$
t_{i j}(\xi)=I+\xi e_{i j}, \quad i \neq j, \quad \xi \in \mathbb{Z} .
$$

The proof of the main result is constructive; in each case the corresponding pairs of $(2,3)$-generators, say $x$ and $y$, are given explicitly. It is well known that, for $n \geq 3$, the group $\mathrm{SL}(n, \mathbb{Z})$ is generated by the transvections

$$
t_{i j}(1), \quad 1 \leq i \neq j \leq n
$$

see [3. The main idea of the proof of Theorem 1.1 is to find enough transvections within the group $\langle x, y\rangle$. The required calculations can be reduced by using the following well-known commutator identity:

$$
t_{i j}(\xi) t_{j k}(\zeta) t_{i j}(\xi)^{-1} t_{j k}(\zeta)^{-1}=t_{i j}(\xi) t_{j k}(\zeta) t_{i j}(-\xi) t_{j k}(-\zeta)=t_{i k}(\xi \zeta)
$$

which is valid for any pairwise distinct $i, j$, and $k$. 
As the following easy lemma shows, for odd $n$ the groups $\operatorname{SL}(n, \mathbb{Z})$ and $\operatorname{GL}(n, \mathbb{Z})$ can be considered simultaneously.

Lemma 2.1. Let $n$ be odd. Let $x, y \in \mathrm{GL}(n, \mathbb{Z})$ and $x^{2}=y^{3}=I$. We have $\langle x, y\rangle=$ $\mathrm{GL}(n, \mathbb{Z})$ if and only if $\langle-x, y\rangle=\mathrm{SL}(n, \mathbb{Z})$.

Proof. Clearly $\operatorname{det} y=1$ and $\operatorname{det} x=-\operatorname{det}(-x)$.

Assume that $\langle x, y\rangle=\mathrm{GL}(n, \mathbb{Z})$. Then $\operatorname{det} x=-1$. Consequently, $\langle-x, y\rangle \subseteq \operatorname{SL}(n, \mathbb{Z})$. It is well known that the derived subgroup of $\mathrm{GL}(n, \mathbb{Z})$ is $\mathrm{SL}(n, \mathbb{Z})$, i.e., any matrix $g \in \mathrm{SL}(n, \mathbb{Z})$ can be represented in the form

$$
g=\prod_{i=1}^{m}\left[g_{2 i-1}(x, y), g_{2 i}(x, y)\right],
$$

where $g_{1}, \ldots, g_{2 m}$ are some words in the alphabet $x, y, y^{-1}$. For any $j$, we have $g_{j}(x, y)=$ $\pm g_{j}(-x, y)$. Therefore,

$$
\left[g_{2 i-1}(x, y), g_{2 i}(x, y)\right]=\left[g_{2 i-1}(-x, y), g_{2 i}(-x, y)\right],
$$

and the inclusion $\operatorname{SL}(n, \mathbb{Z}) \subseteq\langle-x, y\rangle$ follows.

Now assume that $\langle-x, y\rangle=\mathrm{SL}(n, \mathbb{Z})$. The group $\mathrm{SL}(n, \mathbb{Z})$ coincides with its derived subgroup. Hence, any matrix $g \in \mathrm{SL}(n, \mathbb{Z})$ can be represented in the form

$$
g=\prod_{i=1}^{m}\left[g_{2 i-1}(-x, y), g_{2 i}(-x, y)\right]=\prod_{i=1}^{m}\left[g_{2 i-1}(x, y), g_{2 i}(x, y)\right]
$$

where $g_{1}, \ldots, g_{2 m}$ are some words in $x, y, y^{-1}$. Therefore, $\langle x, y\rangle$ contains $\operatorname{SL}(n, \mathbb{Z})$. Since $-x \in \mathrm{SL}(n, \mathbb{Z})$ and $n$ is odd, we have $\operatorname{det} x=-1$. Hence, $\langle x, y\rangle=\operatorname{GL}(n, \mathbb{Z})$.

To prove Theorem 1.2, we also need the following condition sufficient for the group $\langle x, y\rangle$ to be a proper subgroup of $\mathrm{SL}(n, \mathbb{Z})$ or $\mathrm{GL}(n, \mathbb{Z})$.

Lemma 2.2. Let $x, y \in \mathrm{SL}(n, \mathbb{Z}), x^{2}=y^{3}=I$. Assume that there exists a set $\mathcal{W} \subseteq \mathbb{R}^{n}$ and a vector $u \in \mathbb{R}^{n} \backslash \mathcal{W}$ such that

(i) $x y \mathcal{W} \subseteq \mathcal{W}, x y^{2} \mathcal{W} \subseteq \mathcal{W}$;

(ii) $x y u \in \mathcal{W}, x y^{2} u \in \mathcal{W}$.

Then $\langle x, y\rangle \simeq \operatorname{PSL}(2, \mathbb{Z})$. In particular, $\langle x, y\rangle \neq \mathrm{GL}(n, \mathbb{Z}),\langle x, y\rangle \neq \mathrm{SL}(n, \mathbb{Z})$.

Proof. Since $\operatorname{PSL}(2, \mathbb{Z}) \simeq C_{2} * C_{3}$, it suffices to show that the group $\langle x, y\rangle$ is the free product of two cyclic groups of orders 2 and 3 . In other words, we must show that there are no (nontrivial) relations between $x$ and $y$ except $x^{2}=y^{3}=I$.

Let $g$ be a reduced word in the alphabet $x, y$ such that $g=I$. Reducibility means that $g$ does not contain substrings $x x$ and $y y y$.

The assumptions imply that $x \neq I$ and $y \neq I$. Therefore, if $g$ is nonempty, then it contains both $x$ and $y$. Replacing $g$ by a conjugate word (if necessary), we may assume without loss of generality that $g$ is cyclically reduced, i.e., $g$ starts with $x$ and ends with $y$ or $y^{2}$ or $g$ starts with $y$ or $y^{2}$ and ends with $x$. Passing to a conjugate word (if necessary), again we may assume that the first alternative holds; namely,

$$
g=\prod_{i=1}^{m} x y^{\varepsilon_{i}}
$$

where $\varepsilon_{i} \in\{1,2\}$. If $m>0$, then the assumptions of the lemma show that $g u \in \mathcal{W}$, whence $g u \neq u$ and $g \neq I$. This proves that there are no nontrivial relations between $x$ and $y$. 


\section{§3. $n=5$ : PRELIMINARY REMARKS}

The problem on $(2,3)$-generation of the group $\mathrm{GL}(5, \mathbb{Z})$ was considered for the first time by Luzgarëv and Pevzner [5]. They proved the following reduction theorem.

Theorem 3.1 (see [5]). Assume that the group $\mathrm{GL}(5, \mathbb{Z})$ is $(2,3)$-generated. Then the corresponding generating involution and element of order 3 are simultaneously conjugate to

$$
X=\left(\begin{array}{rrrrr}
-1 & 0 & 0 & 0 & 0 \\
0 & -1 & 0 & 0 & 0 \\
0 & 0 & -1 & 0 & 0 \\
1 & 0 & 0 & 1 & 0 \\
0 & 0 & 1 & 0 & 1
\end{array}\right), \quad Y=\left(\begin{array}{rrrrr}
0 & 1 & 0 & 0 & a_{1} \\
-1 & -1 & 0 & 0 & a_{2} \\
0 & 0 & 0 & 1 & a_{3} \\
0 & 0 & -1 & -1 & a_{4} \\
0 & 0 & 0 & 0 & 1
\end{array}\right)
$$

where $\left(a_{1}, a_{2}, a_{3}, a_{4}\right)$ is one of the following sets:

$$
\begin{aligned}
(1,-1,-2,-2), & (0,-1,-2,-2), \\
(1,-1,-2,4), & (0,-1,4,-8), \\
(-1,1,-2,-2), & (0,1,-2,-2), \\
(-1,1,-2,4), & (0,1,4,-8), \\
(1,-1,1,-3), & (0,-1,0,-1) .
\end{aligned}
$$

However, the paper [5] gave no final answer as to whether the group $\operatorname{GL}(5, \mathbb{Z})$ is $(2,3)$-generated.

Remark 3.1. In fact, as was already observed in [5], it suffices to consider only 5 cases out of 10. Namely, two pairs $X, Y$ and $X, Y^{2}$ generate or do not generate $\mathrm{GL}(5, \mathbb{Z})$ simultaneously. The sets in the statement of Theorem 3.1 are grouped into pairs as follows. If a pair $X, Y$ corresponds to the first set in some line (3)-(7), then the second set in the same line corresponds to a pair of matrices that are simultaneously conjugate to $X, Y^{2}$. Thus, it suffices to consider only one quadruple in each line.

It turns out that, in the cases of (3), (5), or (77), the corresponding matrices generate GL $(5, \mathbb{Z})$; see Theorems 4.1, 5.1, and 6.1 below. In this section we concentrate on negative results and show that in the cases of (4) and (6), the matrices $X$ and $Y$ generate proper subgroups of $\mathrm{GL}(5, \mathbb{Z})$.

Theorem 3.2. Let $X, Y$ be defined by (2) with $a_{1}=1, a_{2}=-1, a_{3}=-2$, and $a_{4}=4$. Then $\langle X, Y\rangle \simeq \operatorname{PSL}(2, \mathbb{Z}),\langle-X, Y\rangle \simeq \operatorname{PSL}(2, \mathbb{Z})$.

Proof. We shall apply Lemma 2.2. We start with the following auxiliary construction. Consider the vectors

$$
\begin{aligned}
& v_{1}=(0,0,2,-1,-2)^{t}, \\
& v_{2}=-X Y v_{1}=(-2,2,3,11,-1)^{t}, \\
& v_{3}=(-2,2,1,7,1)^{t}, \\
& v_{4}=-X Y v_{3}=(3,-1,5,1,-6)^{t}, \\
& v_{5}=X Y^{2} v_{3}=(0,3,6,3,-5)^{t}, \\
& v_{6}=-X Y v_{4}=(-7,4,13,37,-7)^{t}, \\
& v_{7}=X Y^{2} v_{4}=(2,-9,18,-9,-24)^{t}, \\
& v_{8}=-X Y v_{5}=(-2,2,13,31,-8)^{t}, \\
& v_{9}=X Y^{2} v_{7}=(-7,-26,57,-23,-81)^{t},
\end{aligned}
$$




$$
\begin{aligned}
& v_{10}=(-1,-1,3,-3,-5)^{t}, \\
& v_{11}=X Y^{2} v_{10}=(-2,-4,10,-5,-15)^{t}, \\
& v_{12}=(0,0,1,2,-2)^{t}
\end{aligned}
$$

where $t$ means transposition. Let

$$
\mathcal{W}_{0}=\operatorname{conv}\left\{\lambda v_{i}: \lambda>0, i=1, \ldots, 12\right\}
$$

be the convex hull of the vectors $\lambda v_{i}, \lambda>0, i=1, \ldots, 12$.

Lemma 3.1. We have $\left(X Y^{2}\right) \mathcal{W}_{0} \subseteq \mathcal{W}_{0}$ and $(-X Y) \mathcal{W}_{0} \subseteq \mathcal{W}_{0}$.

Proof. The proof is based on a direct calculation that employs the convexity of the set $\mathcal{W}_{0}$. For the reader's convenience, the relevant numerical data is presented. It suffices to check that the images of $v_{i}$ under the actions of $X Y^{2}$ and $-X Y$ lie in $\mathcal{W}_{0}$. From the definition of the vectors $v_{1}, \ldots, v_{12}$, it readily follows that

$$
\begin{aligned}
-X Y v_{i} & \in \mathcal{W}_{0} \text { for } i=1,3,4,5, \\
X Y^{2} v_{i} & \in \mathcal{W}_{0} \text { for } i=3,4,7,10 .
\end{aligned}
$$

The remaining inclusions follow from the relations

$$
\begin{aligned}
& X Y^{2} v_{1}=\frac{25}{123} v_{1}+\frac{20}{123} v_{7}+\frac{14}{123} v_{10}+\frac{13}{123} v_{11}+\frac{11}{41} v_{12}, \\
& -X Y v_{2}=v_{1}+\frac{2}{3} v_{3}+v_{4}+\frac{1}{3} v_{8}+v_{12}, \\
& X Y^{2} v_{2}=\frac{241}{67} v_{1}+\frac{11}{67} v_{2}+\frac{48}{67} v_{5}+\frac{11}{67} v_{7}+\frac{71}{67} v_{12}, \\
& X Y^{2} v_{5}=\frac{103}{44} v_{1}+\frac{49}{44} v_{4}+\frac{7}{22} v_{7}+\frac{41}{44} v_{10}+\frac{1}{44} v_{11}, \\
& -X Y v_{6}=\frac{403}{46} v_{3}+\frac{127}{23} v_{4}+\frac{8}{23} v_{8}+\frac{31}{46} v_{11}+\frac{155}{46} v_{12}, \\
& X Y^{2} v_{6}=\frac{93}{4} v_{1}+\frac{3}{10} v_{3}+\frac{9}{20} v_{8}+\frac{3}{2} v_{10}+\frac{137}{20} v_{12} \text {, } \\
& -X Y v_{7}=\frac{11}{5} v_{1}+\frac{24}{5} v_{2}+\frac{56}{5} v_{3}+v_{10}+6 v_{12} \text {, } \\
& -X Y v_{8}=3 v_{1}+3 v_{2}+\frac{14}{5} v_{4}+\frac{6}{5} v_{6}+\frac{12}{5} v_{12}, \\
& X Y^{2} v_{8}=\frac{147}{13} v_{1}+\frac{18}{13} v_{3}+\frac{16}{13} v_{5}+\frac{18}{13} v_{7}+\frac{48}{13} v_{12}, \\
& -X Y v_{9}=\frac{29}{2} v_{2}+\frac{373}{10} v_{3}+\frac{23}{5} v_{5}+\frac{17}{5} v_{10}+\frac{102}{5} v_{12} \text {, } \\
& X Y^{2} v_{9}=\frac{17}{3} v_{1}+\frac{1}{3} v_{2}+\frac{1}{3} v_{6}+\frac{4}{3} v_{9}+\frac{31}{3} v_{11}, \\
& -X Y v_{10}=\frac{813}{256} v_{3}+\frac{1}{8} v_{4}+\frac{35}{128} v_{5}+\frac{3}{256} v_{11}+\frac{369}{256} v_{12} \text {, } \\
& -X Y v_{11}=\frac{3}{2} v_{1}+\frac{23}{12} v_{2}+\frac{91}{12} v_{3}+\frac{2}{3} v_{5}+\frac{14}{3} v_{12}, \\
& X Y^{2} v_{11}=\frac{13}{25} v_{1}+\frac{1}{25} v_{2}+\frac{2}{5} v_{3}+\frac{7}{25} v_{7}+\frac{71}{25} v_{11}, \\
& -X Y v_{12}=v_{3}+\frac{11}{25} v_{4}+\frac{4}{25} v_{6}+\frac{1}{5} v_{10}+\frac{3}{25} v_{12}, \\
& X Y^{2} v_{12}=\frac{148}{123} v_{1}+\frac{20}{123} v_{7}+\frac{14}{123} v_{10}+\frac{13}{123} v_{11}+\frac{11}{41} v_{12} \text {. }
\end{aligned}
$$


Consider the set $\mathcal{W}=\mathcal{W}_{0} \cup\left(-\mathcal{W}_{0}\right)$. By Lemma 3.1, we have

$$
\begin{aligned}
& X Y \mathcal{W} \subseteq \mathcal{W}, \quad X Y^{2} \mathcal{W} \subseteq \mathcal{W}, \\
&-X Y \mathcal{W} \subseteq \mathcal{W}, \quad-X Y^{2} \mathcal{W} \subseteq \mathcal{W} .
\end{aligned}
$$

Take $v=(0,1,2,0,-2)^{t}$ and $u=(-1,2,-2,17,-1)^{t}$. A direct calculation shows that $v^{t} v_{i}>0, i=1, \ldots, 12$. Therefore, $v^{t} w>0$ for any $w \in \mathcal{W}_{0}$, and $v^{t} w<0$ for any $w \in-\mathcal{W}_{0}$. But $v^{t} u=0$. Hence, $u \notin \mathcal{W}$. On the other hand,

$$
\begin{aligned}
X Y u & =-\frac{1}{3} v_{1}-\frac{5}{6} v_{2}-\frac{3}{2} v_{3}-\frac{5}{3} v_{4}-\frac{1}{3} v_{7} \in-\mathcal{W}_{0} \subseteq \mathcal{W}, \\
X Y^{2} u & =\frac{153}{28} v_{1}+\frac{13}{28} v_{4}+\frac{5}{14} v_{5}+\frac{5}{28} v_{10}+\frac{3}{28} v_{11} \in \mathcal{W}_{0} \subseteq \mathcal{W},
\end{aligned}
$$

and in a similar way we obtain

$$
-X Y u \in \mathcal{W}, \quad-X Y^{2} u \in \mathcal{W} .
$$

Relations (8), (10), (11), and Lemma 2.2 applied to $X, Y$ show that $\langle X, Y\rangle \simeq \operatorname{PSL}(2, \mathbb{Z})$. Similarly, (9), (12), and Lemma 2.2 applied to $-X, Y$ imply $\langle-X, Y\rangle \simeq \operatorname{PSL}(2, \mathbb{Z})$.

Theorem 3.3. Let $X, Y$ be defined by (2) with $a_{1}=-1, a_{2}=1, a_{3}=-2, a_{4}=4$. Then $\langle X, Y\rangle \simeq \operatorname{PSL}(2, \mathbb{Z}),\langle-X, Y\rangle \simeq \operatorname{PSL}(2, \mathbb{Z})$.

Proof. The proof is similar to the proof of Theorem 3.2. Consider

$$
\begin{aligned}
v_{1} & =(-1,-1,-1,3,-2)^{t}, \\
v_{2} & =-X Y v_{1}=(1,0,7,9,-5)^{t}, \\
v_{3} & =X Y^{2} v_{1}=(-2,3,6,-3,-8)^{t}, \\
v_{4} & =X Y^{2} v_{3}=(1,10,19,-11,-27)^{t}, \\
v_{5} & =(0,0,1,-1,-1)^{t}, \\
v_{6} & =(2,-2,1,4,1)^{t}, \\
v_{7} & =-X Y v_{6}=(-3,1,2,4,-3)^{t}, \\
v_{8} & =X Y^{2} v_{6}=(0,-3,3,3,-2)^{t}, \\
v_{9} & =-X Y v_{7}=(4,-1,10,14,-7)^{t}, \\
v_{10} & =X Y^{2} v_{7}=(-2,6,12,-2,-15)^{t}, \\
v_{11} & =-X Y v_{8}=(-1,1,7,15,-5)^{t}, \\
v_{12} & =(1,2,4,-4,-6)^{t}, \\
v_{13} & =X Y^{2} v_{12}=(3,5,12,-11,-18)^{t} .
\end{aligned}
$$

Let

$$
\mathcal{W}_{0}=\operatorname{conv}\left\{\lambda v_{i}: \lambda>0, i=1, \ldots, 13\right\}
$$

be the convex hull of the vectors $\lambda v_{i}, \lambda>0, i=1, \ldots, 13$.

Lemma 3.2. We have $\left(X Y^{2}\right) \mathcal{W}_{0} \subseteq \mathcal{W}_{0}$ and $(-X Y) \mathcal{W}_{0} \subseteq \mathcal{W}_{0}$.

Proof. The definitions of the vectors $v_{1}, \ldots, v_{13}$ imply immediately that

$$
\begin{aligned}
& -X Y v_{i} \in \mathcal{W}_{0} \quad \text { for } \quad i=1,6,7,8, \\
& X Y^{2} v_{i} \in \mathcal{W}_{0} \quad \text { for } \quad i=1,3,6,7,12 \text {. }
\end{aligned}
$$


The remaining inclusions follow from the relations

$$
\begin{aligned}
& -X Y v_{2}=\frac{5}{4} v_{1}+\frac{9}{4} v_{2}+\frac{7}{4} v_{5}+2 v_{6}+\frac{1}{4} v_{8}, \\
& X Y^{2} v_{2}=\frac{8}{47} v_{3}+\frac{86}{47} v_{8}+\frac{6}{47} v_{9}+\frac{35}{47} v_{10}+\frac{109}{47} v_{12} \text {, } \\
& -X Y v_{3}=\frac{26}{61} v_{1}+\frac{20}{61} v_{4}+\frac{377}{61} v_{6}+\frac{25}{61} v_{7}+\frac{1}{61} v_{10}, \\
& -X Y v_{4}=\frac{215}{63} v_{1}+\frac{167}{126} v_{2}+\frac{1555}{126} v_{5}+\frac{1184}{63} v_{6}+\frac{3}{2} v_{12} \text {, } \\
& X Y^{2} v_{4}=\frac{1}{2} v_{2}+\frac{5}{2} v_{5}+v_{7}+\frac{15}{2} v_{12}+2 v_{13}, \\
& -X Y v_{5}=\frac{27}{641} v_{1}+\frac{10}{641} v_{5}+\frac{310}{641} v_{6}+\frac{18}{641} v_{9}+\frac{24}{641} v_{11} \text {, } \\
& X Y^{2} v_{5}=\frac{1}{24} v_{1}+\frac{1}{12} v_{4}+\frac{1}{24} v_{5}+\frac{1}{24} v_{7}+\frac{1}{12} v_{12} \text {, } \\
& X Y^{2} v_{8}=\frac{1}{8} v_{1}+\frac{11}{8} v_{5}+\frac{5}{8} v_{7}+\frac{1}{2} v_{8}+\frac{1}{2} v_{10} \text {, } \\
& -X Y v_{9}=\frac{92}{41} v_{1}+\frac{426}{41} v_{5}+\frac{413}{82} v_{6}+\frac{165}{82} v_{11}+\frac{5}{82} v_{13}, \\
& X Y^{2} v_{9}=v_{4}+\frac{5}{2} v_{5}+\frac{19}{6} v_{8}+\frac{1}{6} v_{9}+\frac{4}{3} v_{12}, \\
& -X Y v_{10}=\frac{47}{27} v_{1}+\frac{40}{27} v_{2}+\frac{110}{27} v_{5}+\frac{269}{27} v_{6}+\frac{4}{3} v_{12}, \\
& X Y^{2} v_{10}=\frac{1}{2} v_{2}+\frac{2}{3} v_{3}+\frac{5}{6} v_{4}+\frac{2}{3} v_{5}+\frac{4}{3} v_{13} \text {, } \\
& -X Y v_{11}=\frac{61}{42} v_{1}+\frac{101}{42} v_{2}+\frac{65}{21} v_{5}+\frac{32}{21} v_{6}+\frac{1}{2} v_{9}, \\
& X Y^{2} v_{11}=\frac{15}{43} v_{1}+\frac{523}{43} v_{5}+\frac{20}{43} v_{7}+\frac{48}{43} v_{11}+\frac{41}{43} v_{13} \text {, } \\
& -X Y v_{12}=\frac{1}{2} v_{1}+v_{5}+4 v_{6}+\frac{1}{2} v_{8}+\frac{1}{2} v_{12}, \\
& -X Y v_{13}=2 v_{1}+\frac{620}{71} v_{5}+\frac{941}{71} v_{6}+\frac{56}{71} v_{7}+\frac{61}{71} v_{12} \text {, } \\
& X Y^{2} v_{13}=\frac{1}{6} v_{1}+\frac{1}{4} v_{3}+\frac{1}{3} v_{8}+\frac{35}{12} v_{12}+\frac{23}{12} v_{13} \text {. }
\end{aligned}
$$

Set $\mathcal{W}=\mathcal{W}_{0} \cup\left(-\mathcal{W}_{0}\right)$. By Lemma 3.2, we have

$$
\begin{aligned}
& X Y \mathcal{W} \subseteq \mathcal{W}, \quad X Y^{2} \mathcal{W} \subseteq \mathcal{W}, \\
&-X Y \mathcal{W} \subseteq \mathcal{W}, \quad-X Y^{2} \mathcal{W} \subseteq \mathcal{W} .
\end{aligned}
$$

Take $v=(0,0,2,1,0)^{t}$ and $u=(0,0,-1,2,0)^{t}$. A direct calculation shows that $v^{t} v_{i}>0$ for all $i=1, \ldots, 13$. Therefore, $v^{t} w \neq 0$ for any $w \in \mathcal{W}$. On the other hand, $v^{t} u=0$, Hence, $u \neq \mathcal{W}$. Moreover,

$$
\begin{aligned}
X Y u & =-\frac{1}{11} v_{1}-\frac{5}{44} v_{2}-\frac{39}{44} v_{5}-\frac{1}{22} v_{11}-\frac{1}{44} v_{12} \in-\mathcal{W}_{0} \subseteq \mathcal{W}, \\
X Y^{2} u & =v_{5} \in \mathcal{W}_{0} \subseteq \mathcal{W}, \\
-X Y u & \in \mathcal{W}, \quad-X Y^{2} u \in \mathcal{W} .
\end{aligned}
$$

To complete the proof, we apply Lemma 2.2 just as we did in Theorem 3.2 . 


\section{§4. $n=5$ : The FIRSt Generating SET}

In this section we consider the case where $X$ and $Y$ are given by (2) with $a_{1}=1$, $a_{2}=-1, a_{3}=-2$, and $a_{4}=-2$. For technical reasons, it is more convenient to work not with the matrices $X$ and $Y$ but rather with their conjugates. Let

$$
Q=\left(\begin{array}{rrrrr}
1 & 0 & 0 & 0 & 0 \\
0 & 1 & 1 & -1 & 1 \\
0 & 2 & 0 & -1 & 0 \\
0 & 0 & 1 & 2 & 3 \\
0 & 3 & 0 & 0 & 1
\end{array}\right)
$$

We set

$$
\begin{array}{r}
x=Q X Q^{-1}=\left(\begin{array}{rrrrr}
-1 & 0 & 0 & 0 & 0 \\
-1 & -6 & 19 & 6 & -11 \\
-1 & -4 & 13 & 4 & -8 \\
2 & 5 & -13 & -3 & 7 \\
0 & -1 & 5 & 2 & -4
\end{array}\right), \\
y=Q Y Q^{-1}=\left(\begin{array}{rrrrr}
0 & -2 & 6 & 2 & -3 \\
-1 & 8 & -24 & -7 & 13 \\
-2 & 5 & -14 & -4 & 7 \\
0 & -3 & 6 & 1 & -3 \\
-3 & 3 & -9 & -3 & 4
\end{array}\right) .
\end{array}
$$

Theorem 4.1. Let $x, y$ be given by (13), (14). Then $\langle x, y\rangle=\mathrm{GL}(5, \mathbb{Z})$ and $\langle-x, y\rangle=$ $\mathrm{SL}(5, \mathbb{Z})$.

The key step of the proof is the following lemma.

Lemma 4.1. Let $G$ be a subgroup of $\mathrm{GL}(5, \mathbb{Z})$ containing $x, y$, and $t_{51}(a)$ for some $a \in \mathbb{Z}$. Then $G$ also contains all the transvections $t_{i j}(a)$ with $1 \leq i \neq j \leq 5$.

Proof. In what follows, the elements of $G$ that do not depend on $a$ will be denoted by $h$ with subscripts, while the elements that do depend on $a$ will be denoted by $z$ with subscripts. Let

$$
z_{1}:=t_{51}(a) .
$$

We set

$$
\begin{aligned}
& h_{1}:=\left(\left(x y x y^{2}\right)^{2}(x y)^{3} x\right)^{6}, \\
& h_{2}:=x y x y^{2}(x y)^{5} x y^{2}, \\
& h_{3}:=x y^{2} x y x, \\
& h_{4}:=h_{2} x, \\
& h_{5}:=x y x y^{2} x y\left(x y^{2}\right)^{2}(x y)^{4} x, \\
& h_{6}:=\left(x y^{2}\right)^{3}(x y)^{3}\left(x y^{2}\right)^{2} x y .
\end{aligned}
$$

Next, we define

$$
\begin{aligned}
& z_{2}:=h_{2} z_{1} h_{2}^{-1}=I+a\left(10 e_{13}-16 e_{23}-3 e_{43}-2 e_{53}\right), \\
& z_{3}:=h_{4} z_{1} h_{4}^{-1}=I+a\left(e_{13}-3 e_{23}+4 e_{43}+3 e_{53}\right), \\
& z_{4}:=h_{3} h_{4} z_{1} h_{4}^{-1} h_{3}^{-1}=I+a\left(e_{13}-3 e_{23}-7 e_{43}-2 e_{53}\right), \\
& z_{5}:=h_{2} h_{5} h_{1} h_{5}^{-1} z_{1} h_{5} h_{1}^{-1} h_{5}^{-1} h_{2}^{-1}=I+a\left(-8 e_{13}+11 e_{23}+6 e_{43}+4 e_{53}\right), \\
& z_{6}:=h_{6} h_{3} h_{6}^{-1} h_{4} z_{1} h_{4}^{-1} h_{6} h_{3}^{-1} h_{6}^{-1}=I+a\left(13 e_{13}-17 e_{23}-32 e_{43}+3 e_{53}\right) .
\end{aligned}
$$


It is clear that the elements $z_{2}, \ldots, z_{6}$ commute pairwise; we can use them for finding an elementary transvection of level $a$. Namely,

$$
\begin{aligned}
z_{7} & :=z_{2}^{-565} z_{3}^{617} z_{4}^{151} z_{5}^{-651} z_{6}^{-25}=t_{13}(a), \\
z_{8} & :=z_{2}^{-46} z_{3}^{50} z_{4}^{12} z_{5}^{-53} z_{6}^{-2}=t_{23}(a), \\
z_{9} & :=z_{2}^{-1059} z_{3}^{1157} z_{4}^{284} z_{5}^{-1220} z_{6}^{-47}=t_{43}(a), \\
z_{10} & :=z_{2}^{585} z_{3}^{-639} z_{4}^{-157} z_{5}^{674} z_{6}^{26}=t_{53}(a) .
\end{aligned}
$$

Next, we introduce

$$
\begin{aligned}
& z_{11}:=h_{2}^{-1} z_{7}^{5} z_{8}^{-7} z_{9}^{-3} z_{10}^{-2} h_{2}=t_{21}(a), \\
& z_{12}:=h_{2}^{-1} z_{7}^{-17} z_{8}^{26} z_{9}^{7} z_{10}^{5} h_{2}=t_{31}(a), \\
& z_{13}:=h_{2}^{-1} z_{7}^{-6} z_{8}^{10} z_{9} z_{10} h_{2}=t_{41}(a) .
\end{aligned}
$$

Now, we set

$$
\begin{aligned}
h_{7} & :=x y\left(x y^{2}\right)^{2} x y x y^{2}(x y)^{2} x y^{2} x, \\
h_{8} & :=x y^{2}(x y)^{2} x y^{2} x y x y^{2}(x y)^{3} x, \\
h_{9} & :=x y^{2}(x y)^{2}\left(x y^{2}\right)^{2} x y x, \\
h_{10} & :=(x y)^{3}\left(x y^{2}\right)^{2}
\end{aligned}
$$

and consider

$$
\begin{aligned}
& z_{14}:=h_{7} z_{7} h_{7}^{-1}=I+a\left(-2 e_{12}-2 e_{14}+e_{15}\right), \\
& z_{15}:=h_{8} z_{7} h_{8}^{-1}=I+a\left(-3 e_{12}+9 e_{13}+3 e_{14}-4 e_{15}\right), \\
& z_{16}:=h_{9} z_{7} h_{9}^{-1}=I+a\left(5 e_{12}-17 e_{13}-5 e_{14}+8 e_{15}\right), \\
& z_{17}:=h_{10} z_{7} h_{10}^{-1}=I+a\left(-9 e_{12}+31 e_{13}+8 e_{14}-16 e_{15}\right) .
\end{aligned}
$$

The matrices $z_{7}, z_{14}, z_{15}, z_{16}$, and $z_{17}$ commute pairwise, and

$$
\begin{aligned}
& z_{18}:=z_{7}^{-1} z_{15}^{-4} z_{16}^{-4} z_{17}^{-1}=t_{12}(a), \\
& z_{19}:=z_{7}^{-2} z_{15}^{-2} z_{16}^{-3} z_{17}^{-1}=t_{14}(a), \\
& z_{20}:=z_{7}^{-6} z_{14} z_{15}^{-12} z_{16}^{-14} z_{17}^{-4}=t_{15}(a) .
\end{aligned}
$$

In a similar way, let

$$
\begin{aligned}
& h_{11}:=x y x y^{2} x y x \\
& h_{12}:=\left(x y^{2}\right)^{3} x y x \\
& h_{13}:=(x y)^{2} x y^{2}(x y)^{3} x, \\
& z_{21}:=h_{11} z_{7} h_{11}^{-1}=I+a\left(2 e_{41}+3 e_{42}-3 e_{43}+e_{45}\right), \\
& z_{22}:=h_{12} z_{7} h_{12}^{-1}=I+a\left(-2 e_{42}+e_{45}\right), \\
& z_{23}:=h_{13} z_{7} h_{13}^{-1}=I+a\left(-2 e_{41}-e_{42}+e_{43}-e_{45}\right) .
\end{aligned}
$$

Using these matrices, we find

$$
\begin{aligned}
& z_{24}:=z_{9}^{2} z_{21} z_{22} z_{23}=t_{45}(a), \\
& z_{25}:=z_{9}^{-1} z_{13}^{-2} z_{21}^{-1} z_{22}^{-1} z_{23}^{-2}=t_{42}(a) .
\end{aligned}
$$


Finally, consider

$$
\begin{aligned}
& h_{14}:=x y x y^{2}, \\
& z_{26}:=h_{14} z_{19} h_{14}^{-1} z_{18} z_{25}^{-2}=t_{52}(a), \\
& h_{15}:=\left(x y x y^{2}\right)^{3} x \\
& z_{27}:=h_{15} z_{25}^{-1} h_{15}^{-1} z_{1}^{-6} z_{12}^{-3} z_{26}^{-2}=t_{32}(a), \\
& z_{28}:=h_{15} z_{10}^{-1} h_{15}^{-1} z_{26}^{-1}=t_{54}(a), \\
& z_{29}:=h_{15} z_{9} h_{15}^{-1} z_{26}^{-2} z_{27}^{-1} z_{28}^{-2}=t_{34}(a), \\
& z_{30}:=h_{14}^{-1} z_{18}^{-1} z_{25}^{2} z_{26}^{4} z_{27}^{2} h_{14}=t_{24}(a), \\
& h_{16}:=x y x y^{2}(x y)^{2} x y^{2} x, \\
& z_{31}:=h_{16} z_{8}^{-1} h_{16}^{-1} z_{11}^{-1}=t_{25}(a), \\
& h_{17}:=x y x y^{2}(x y)^{3}\left(x y^{2}\right)^{2}(x y)^{2}\left(x y^{2}\right)^{2}(x y)^{2}\left(x y^{2}\right)^{2}, \\
& h_{18}:=h_{17} x h_{17}^{-1}, \\
& z_{32}:=h_{18} z_{12} h_{18} z_{12} z_{27} z_{29}=t_{35}(a) .
\end{aligned}
$$

The assumption that $t_{51}(a), x, y \in G$, and relations (17)-(35) imply that $G$ contains all elementary transvections $t_{i j}(a)$ of level $a, 1 \leq i \neq j \leq 5$.

Now we are ready to prove Theorem 4.1

Proof of Theorem 4.1. We keep the notation used in the proof of Lemma 4.1. First, observe that the matrix $h_{1}$ defined by (15) is an elementary transvection of level 3 :

$$
h_{1}=t_{51}(3) \text {. }
$$

By Lemma 4.1, $\langle x, y\rangle$ contains $t_{i j}(3)$ for all $1 \leq i \neq j \leq 5$.

Also, we note that the matrix $h_{3}$ defined by (16) has a rather simple shape:

$$
h_{3}=\left(\begin{array}{rrrrr}
-1 & 0 & 0 & 0 & 0 \\
0 & -1 & 0 & 0 & 0 \\
0 & 0 & -1 & 0 & 0 \\
3 & 0 & 1 & 1 & 0 \\
2 & 1 & -3 & 0 & 1
\end{array}\right) .
$$

Our next aim is to find other matrices of the same block-triangular shape. Set

$$
h_{19}:=\left(y x y^{2} x\right)^{2}\left(y^{2} x y x\right)\left(y^{2} x\right)^{3} y x y^{2} x(y x)^{4}\left(y^{2} x\right)^{2} y x y^{2} x y .
$$

The matrix $h_{19}$ modulo 3 is a block-triangular matrix. This suggests that we can find block-triangular matrices by using $h_{19}$ and transvections of level 3. Namely, let

$$
\begin{aligned}
& h_{20}:=h_{19} \cdot\left(t_{14}(3)\right)^{-239}\left(t_{24}(3)\right)^{704}\left(t_{34}(3)\right)^{189}\left(t_{15}(3)\right)^{-229}\left(t_{25}(3)\right)^{673}\left(t_{35}(3)\right)^{181}, \\
& h_{21}:=\left(t_{14}(3)\right)^{2026}\left(t_{15}(3)\right)^{3016}\left(t_{24}(3)\right)^{-1115}\left(t_{25}(3)\right)^{-1661}\left(t_{34}(3)\right)^{26}\left(t_{35}(3)\right)^{38} h_{20} .
\end{aligned}
$$

The matrix $h_{21}$ is block-triangular. Consider

$$
\begin{aligned}
& h_{22}:=h_{3} h_{21} h_{3} h_{21}^{-1}, \\
& h_{23}:=h_{3} h_{21}^{2} h_{3} h_{21}^{-2}, \\
& h_{24}:=h_{3} h_{21}^{3} h_{3} h_{21}^{-3}, \\
& h_{25}:=h_{3} h_{21}^{-2} h_{3} h_{21}^{2} .
\end{aligned}
$$


It is easy to check that

$$
\begin{aligned}
h_{26}:= & h_{22} h_{23} h_{24} h_{25} \\
= & I-39868892529699498 e_{41}-66045130609368789 e_{42} \\
& +276830690835290373 e_{43}+24288462735300964 e_{51} \\
& +40192071970237800 e_{52}-168478035844626270 e_{53}
\end{aligned}
$$

In particular, $h_{26}, t_{41}(3), t_{42}(3), t_{43}(3), t_{51}(3), t_{52}(3)$, and $t_{53}(3)$ commute pairwise, and

$$
\begin{aligned}
h_{27}:= & h_{26} \cdot\left(t_{41}(3)\right)^{13289630843233166} \cdot\left(t_{42}(3)\right)^{22015043536456263} \\
& \times\left(t_{43}(3)\right)^{-92276896945096791} \cdot\left(t_{51}(3)\right)^{-8096154245100321} \\
& \times\left(t_{52}(3)\right)^{-13397357323412600} \cdot\left(t_{53}(3)\right)^{56159345281542090} \\
= & t_{51}(1) .
\end{aligned}
$$

By Lemma 4.1, all the transvections $t_{i j}(1), 1 \leq i \neq j \leq 5$, lie in $\langle x, y\rangle$. Therefore, $\langle x, y\rangle$ contains $\mathrm{SL}(5, \mathbb{Z})$. Since $\langle x, y\rangle$ also contains matrices of determinant -1 (e.g., $x$ ), the group $\langle x, y\rangle$ coincides with $\mathrm{GL}(5, \mathbb{Z})$. Lemma 2.1 implies that $\langle-x, y\rangle=\mathrm{SL}(5, \mathbb{Z})$.

\section{§5. $n=5$ : The SeCOnd Generating SeT}

In this section we consider the matrices $X$ and $Y$ given by (2) with $a_{1}=-1, a_{2}=1$, $a_{3}=-2$, and $a_{4}=-2$. As in the preceding section, it is more convenient to work with conjugates of $X$ and $Y$ instead of $X$ and $Y$ themselves. Put

$$
Q=\left(\begin{array}{rrrrr}
1 & 13 & 2 & 0 & -8 \\
0 & 1 & 0 & 0 & -1 \\
0 & 0 & 1 & 0 & 0 \\
0 & 0 & -1 & 1 & 0 \\
0 & 0 & 0 & 0 & 1
\end{array}\right)
$$

and

$$
\begin{aligned}
& x=Q X Q^{-1}=\left(\begin{array}{rrrrr}
-1 & 0 & -8 & 0 & -16 \\
0 & -1 & -1 & 0 & -2 \\
0 & 0 & -1 & 0 & 0 \\
1 & -13 & 0 & 1 & -5 \\
0 & 0 & 1 & 0 & 1
\end{array}\right), \\
& y=Q Y Q^{-1}=\left(\begin{array}{rrrrr}
-13 & 157 & 28 & 2 & 53 \\
-1 & 12 & 2 & 0 & 4 \\
0 & 0 & 1 & 1 & -2 \\
0 & 0 & -3 & -2 & 0 \\
0 & 0 & 0 & 0 & 1
\end{array}\right) .
\end{aligned}
$$

Theorem 5.1. Let $x$ and $y$ be defined by (36) and (37). Then $\langle x, y\rangle=\mathrm{GL}(5, \mathbb{Z})$ and $\langle-x, y\rangle=\operatorname{SL}(5, \mathbb{Z})$.

We start with the following auxiliary lemma.

Lemma 5.1. Let $G$ be a subgroup of $\mathrm{GL}(5, \mathbb{Z})$ containing $x, y$, and $t_{32}(a)$ for some $a \in \mathbb{Z}$. Then $G$ also contains $t_{12}(a), t_{42}(a)$, and $t_{52}(a)$. 
Proof. As before, by $h$ with subscripts we denote elements of the group $G$ that do not depend on $a$, and by $z$ with subscripts we denote elements that depend on $a$. Set

$$
\begin{aligned}
& h_{1}:=y^{2} x y \\
& h_{2}:=y x y^{2} \\
& h_{3}:=\left(x y^{2}\right)^{3}(x y)^{3} x, \\
& h_{4}:=x y^{2} x y\left(x y^{2}\right)^{3}(x y)^{2} x y^{2}(x y)^{2}\left(x y^{2}\right)^{2} x, \\
& z_{1}:=t_{32}(a)
\end{aligned}
$$

and consider

$$
\begin{aligned}
& z_{2}:=h_{1} z_{1} h_{1}=I+a\left(-5 e_{12}+e_{32}-2 e_{42}-e_{52}\right), \\
& z_{3}:=h_{2} z_{1} h_{2}=I+a\left(-8 e_{12}-7 e_{32}+8 e_{42}+2 e_{52}\right), \\
& z_{4}:=h_{3} h_{1} z_{1} h_{1} h_{3}=I+a\left(-34 e_{12}+65 e_{32}-58 e_{42}-35 e_{52}\right), \\
& z_{5}:=h_{4} h_{1} z_{1} h_{1} h_{4}^{-1}=I+a\left(-138 e_{12}+313 e_{32}-325 e_{42}-161 e_{52}\right) .
\end{aligned}
$$

In particular, the elements $z_{1}, z_{2}, z_{3}, z_{4}$, and $z_{5}$ commute pairwise. Using easy linear algebra, we see that

$$
\begin{aligned}
& z_{6}:=z_{1}^{808} z_{2}^{-6983} z_{3}^{3414} z_{4}^{-1059} z_{5}^{316}=t_{12}(a) \\
& z_{7}:=z_{1}^{146} z_{2}^{-1260} z_{3}^{616} z_{4}^{-191} z_{5}^{57}=t_{42}(a) \\
& z_{8}:=z_{1}^{-341} z_{2}^{2962} z_{3}^{-1448} z_{4}^{449} z_{5}^{-134}=t_{52}(a) .
\end{aligned}
$$

This completes the proof.

Proof of Theorem 5.1. Our first aim is to find sufficiently many matrices of the form

$$
\left(\begin{array}{cc}
I_{2} & 0 \\
* & I_{3}
\end{array}\right) \text {. }
$$

However, we start with slightly more general matrices

$$
\left(\begin{array}{cc}
Q_{1} & 0 \\
Q_{2} & Q_{3}
\end{array}\right),
$$

where $Q_{1}$ and $Q_{3}$ are square matrices of size 2 and 3 , respectively. Consider

$$
\begin{aligned}
h_{5}:=x y\left(x y^{2}\right)^{7}(x y)^{6} & =\left(\begin{array}{rrrrr}
11 & -120 & 0 & 0 & 0 \\
1 & -11 & 0 & 0 & 0 \\
-39 & 532 & -14 & -28 & 45 \\
57 & -773 & 15 & 34 & -60 \\
17 & -232 & 5 & 11 & -19
\end{array}\right), \\
h_{6}:=x y x y^{2} x & =\left(\begin{array}{rrrrr}
-11 & 120 & 0 & 0 & 0 \\
-1 & 11 & 0 & 0 & 0 \\
-3 & 38 & -1 & -2 & 6 \\
5 & -65 & 0 & 4 & -15 \\
2 & -25 & 0 & 1 & -4
\end{array}\right), \\
h_{7}:=\left(y^{2} x\right)^{2} y x y^{2} x y x y & =\left(\begin{array}{rrrrr}
1 & -30 & 0 & 0 & 0 \\
0 & -1 & 0 & 0 & 0 \\
11 & -129 & -49 & -24 & -48 \\
-14 & 165 & 60 & 29 & 60 \\
-4 & 45 & 20 & 10 & 19
\end{array}\right) .
\end{aligned}
$$


TABLE 1

\begin{tabular}{|r|r|r|r|r|r|r|}
\hline & $k_{31}$ & $k_{32}$ & $k_{41}$ & $k_{42}$ & $k_{51}$ & $k_{52}$ \\
\hline$h_{8}$ & 8878 & -88380 & -11492 & 115170 & -3814 & 38190 \\
$h_{9}$ & 5628 & -47130 & -6992 & 58920 & -2314 & 19440 \\
$h_{10}$ & 15504 & -144690 & -17218 & 160680 & -5756 & 53760 \\
$h_{11}$ & 9378 & -94380 & -11492 & 115170 & -3814 & 38190 \\
$h_{12}$ & 23858 & -449160 & -29428 & 554310 & -9826 & 185070 \\
$h_{13}$ & -187422 & 2085600 & 232352 & -2585550 & 77334 & -860550 \\
$h_{14}$ & 1253860 & -13921500 & -1401298 & 15558450 & -467216 & 5187450 \\
$h_{15}$ & -586936 & 7760460 & 657316 & -8691870 & 219582 & -2905870 \\
\hline
\end{tabular}

Now, it is easy to verify that the following matrices have the desired shape (38):

$$
\begin{aligned}
h_{8} & :=\left(h_{5}^{-6} h_{6} h_{7} h_{5}^{-2} h_{7}\right)^{2}, \\
h_{9} & :=\left(h_{7} h_{5}^{-2} h_{7} h_{6}\right)^{2}, \\
h_{10} & :=h_{5} h_{8} h_{5}^{-1} \\
h_{11} & :=h_{6} h_{8} h_{6}^{-1} \\
h_{12} & :=h_{7} h_{8} h_{7}^{-1} \\
h_{13} & :=h_{6} h_{7} h_{8} h_{7}^{-1} h_{6}^{-1}, \\
h_{14} & :=h_{5} h_{7} h_{5} h_{8} h_{5}^{-1} h_{7}^{-1} h_{5}^{-1} \\
h_{15} & :=\left(h_{5} h_{6} h_{7}\left(h_{6} h_{7} h_{6} h_{7} h_{5}^{5}\right)^{2} h_{6} h_{7} h_{6} h_{5}^{3}\right)^{2} .
\end{aligned}
$$

Namely, each of the eight matrices $h_{8}, \ldots, h_{15}$ can be written as

$$
h_{i}=I+k_{31} e_{31}+k_{32} e_{32}+k_{41} e_{41}+k_{42} e_{42}+k_{51} e_{51}+k_{52} e_{52},
$$

where the coefficients $k_{i j}$ are listed in Table 1 . In particular, the matrices $h_{8}, \ldots, h_{15}$ commute pairwise, and we can use their combination in order to find an elementary transvection of level 2:

$$
\begin{aligned}
h_{16}:= & h_{8}^{-177312135} h_{9}^{12142834959} h_{10}^{-3022311905} h_{11}^{-18496419129} \\
& \times h_{12}^{-438573050} h_{13}^{-495339791} h_{14}^{56766741} \\
= & t_{31}(2) .
\end{aligned}
$$

We also need another transvection of level 10. Namely, set

$$
\begin{aligned}
h_{17} & :=h_{8}^{250005876} h_{9}^{-92554481} h_{10}^{5425894} h_{11}^{-47781509} h_{12}^{2141478} h_{13}^{12452569} h_{14}^{577930} h_{15}^{-380735} \\
& =I+30 e_{32}-30 e_{42}-10 e_{52}
\end{aligned}
$$

and consider the following product of conjugates of $h_{17}$ :

$$
h_{18}:=h_{17}^{-510} h_{1} h_{17}^{-2520} h_{1}^{-1} h_{3} h_{17}^{472} h_{3}^{-1} h_{4} h_{17}^{50} h_{4}^{-1} h_{1} h_{4} h_{17}^{-71} h_{4}^{-1} h_{1}^{-1}=t_{32}(10) .
$$

(It is clear that $h_{1}$ is an involution and we can write $h_{1}$ instead of $h_{1}^{-1}$. However, here we prefer to keep the notation $h_{1}^{-1}$, in order to emphasize that the above expression for $h_{18}$ is a product of conjugates of $h_{17}$.)

Now we use suitable conjugates of $h_{16}$ for finding further transvections, Take

$$
\begin{aligned}
& h_{19}:=y^{2}\left(x y^{2}\right)^{5} x y\left(x y^{2}\right)^{2}, \\
& h_{20}:=y x y^{2} x(y x)^{3}\left(y^{2} x\right)^{4} y^{2}, \\
& h_{21}:=y x y^{2} x(y x)^{2} y^{2} x y x\left(y^{2} x\right)^{2}(y x)^{3}\left(y^{2} x\right)^{5}(y x)^{3}\left(y^{2} x\right)^{3} y x y^{2},
\end{aligned}
$$


and set

$$
\begin{aligned}
& h_{22}::=h_{19} h_{16} h_{19}^{-1}, \\
& h_{23}:=h_{20} h_{16} h_{20}^{-1}, \\
& h_{24}:=h_{21} h_{16} h_{21}^{-1}, \\
& h_{25}:=h_{22} h_{16} h_{22}^{-1}=I+3194 e_{31}-40964 e_{32}-3724 e_{34}+9044 e_{35}, \\
& h_{26}:=h_{23} h_{16} h_{23}^{-1}=I+11930 e_{31}-115020 e_{32}+21300 e_{34}-32376 e_{35}, \\
& h_{27}:=h_{24} h_{16} h_{24}^{-1}=I+120283562 e_{31}-1382839856 e_{32} \\
& \quad+152542256 e_{34}-229142928 e_{35} .
\end{aligned}
$$

In particular, $h_{16}, h_{18}, h_{25}, h_{26}$, and $h_{27}$ commute pairwise, and

$$
\begin{aligned}
h_{28} & :=h_{16}^{-124140789441885} h_{18}^{413807222290788} h_{25}^{-6525940705} h_{26}^{-59250292539} h_{27}^{8114005} \\
& =t_{35}(4), \\
h_{29} & :=h_{16}^{-1105501046986} h_{18}^{3685044372190} h_{25}^{-58114938} h_{26}^{-527636893} h_{27}^{72257}=t_{34}(4), \\
h_{30} & :=h_{16}^{68914022536559} h_{18}^{-229715956946515} h_{25}^{3622732116} h_{26}^{32891493712} h_{27}^{-4504311}=t_{32}(2) .
\end{aligned}
$$

By (41), we have $t_{32}(2) \in\langle x, y\rangle$. Lemma 5.1 implies that

$$
\begin{aligned}
h_{31} & :=t_{12}(2), \\
h_{32} & :=t_{42}(2), \\
h_{33} & :=t_{52}(2)
\end{aligned}
$$

are also in $\langle x, y\rangle$. Next, we have

$$
\begin{aligned}
h_{34} & :=h_{8}^{4840} h_{9}^{-2962} h_{10}^{420} h_{11}^{929} h_{12}^{86} h_{13}^{291} h_{14}^{5} h_{15}^{-8} h_{30}^{9150} h_{32}^{8445} h_{33}^{1895}=I+2 e_{41}+4 e_{51}, \\
h_{35} & :=h_{8}^{-3480} h_{9}^{1882} h_{10}^{25} h_{11}^{330} h_{12}^{-12} h_{13}^{-168} h_{14}^{-11} h_{15} h_{30}^{-3585} h_{32}^{-2400} h_{33}^{-7660}=t_{51}(10) .
\end{aligned}
$$

Setting

$$
\begin{aligned}
& h_{36}:=h_{19}^{-1} h_{16} h_{19}, \\
& h_{37}:=h_{20}^{-1} h_{16} h_{20},
\end{aligned}
$$

we see that

$$
\begin{aligned}
& h_{38}:=h_{36} h_{16} h_{36}^{-1}=I+532 e_{21}-11702 e_{31}+11704 e_{41}+4256 e_{51}, \\
& h_{39}:=h_{37} h_{16} h_{37}^{-1}=I-1704 e_{21}-30670 e_{31}+11076 e_{41}+17892 e_{51} .
\end{aligned}
$$

Clearly, $h_{16}, h_{34}, h_{35}, h_{38}$, and $h_{39}$ commute pairwise. By an easy calculation,

$$
\begin{aligned}
h_{40} & :=h_{16}^{9064162} h_{34}^{-6459011} h_{35}^{1745066} h_{38}^{852} h_{39}^{266}=t_{41}(2), \\
h_{41} & :=h_{16}^{-4532081} h_{34}^{3229506} h_{35}^{-872533} h_{38}^{-426} h_{39}^{-133}=t_{51}(2), \\
h_{42} & :=h_{16}^{2180895} h_{34}^{-1554092} h_{35}^{419880} h_{38}^{205} h_{39}^{64}=t_{21}(4) .
\end{aligned}
$$

Set

$$
h_{43}:=y^{2} x(y x)^{2} y^{2} h_{31} y\left(x y^{2}\right)^{2} x y ;
$$

then

$$
h_{44}:=h_{43} h_{31} h_{43}^{-1} h_{31}^{3}=I+4 e_{13}+8 e_{15} .
$$


We take

$$
h_{45}:=\left(h_{5} h_{6} h_{7}\right)^{2}\left(h_{6} h_{7} h_{5}^{-1}\right)^{2} h_{5}^{36}=\left(\begin{array}{rrrrr}
1 & 0 & 0 & 0 & 0 \\
0 & 1 & 0 & 0 & 0 \\
-31227 & 274086 & 1 & 36 & -108 \\
32975 & -286842 & 0 & 1 & 0 \\
11019 & -95904 & 0 & 0 & 1
\end{array}\right) .
$$

It is easily seen that

$$
h_{46}:=h_{45} h_{28}^{27} h_{29}^{-9} h_{16}^{15613} h_{30}^{-137043} h_{32}^{143421} h_{33}^{47952} h_{40}^{-16485} h_{41}^{-5509}=I-e_{31}+5 e_{41}+e_{51} .
$$

(For further purposes, the matrix $h_{46}$ is slightly more convenient than, say, the matrix $I+e_{31}+e_{41}+e_{51}$, which can be obtained in a similar way. For instance, the shape of $x h_{46} x$ is simpler; see also the definition of $h_{47}$ below.) Now we take

$$
\begin{aligned}
h_{47} & :=h_{44}^{2} x h_{46} x h_{44}^{-2}=I+e_{21}-e_{31}, \\
h_{48} & :=h_{16}^{-1} h_{42} h_{47}^{-2}=t_{21}(2), \\
h_{49} & :=h_{31}^{-6} h_{6} h_{47} h_{6}^{-1} h_{31}^{5}\left(h_{48} h_{31}^{-1}\right)^{6} h_{31}^{-5} h_{48} \\
& =I+e_{21}-897 e_{31}+1495 e_{41}+575 e_{51}-468 e_{32}+780 e_{42}+300 e_{52}, \\
h_{50} & :=h_{46} h_{47}^{-1} h_{49} h_{16}^{449} h_{30}^{234} h_{32}^{-390} h_{33}^{-150} h_{40}^{-750} h_{41}^{-288}=t_{31}(1), \\
h_{51} & :=h_{47} h_{50}=t_{21}(1) .
\end{aligned}
$$

The next step is to find other transvections of level 1. Consider

$$
\begin{aligned}
h_{52} & :=(y x)^{3} y^{2} x(y x)^{4} y^{2} x(y x)^{2}\left(y^{2} x\right)^{2}(y x)^{4} y^{2}, \\
h_{53} & :=h_{52} h_{50} h_{52}^{-1}, \\
h_{54} & :=h_{19} h_{50} h_{19}^{-1}, \\
h_{55} & :=h_{20} h_{50} h_{20}^{-1} .
\end{aligned}
$$

We arrive at

$$
\begin{aligned}
& h_{56}:=h_{53} h_{50} h_{53}^{-1}=I-56979 e_{31}+197395 e_{32}+85470 e_{34}+93610 e_{35}, \\
& h_{57}:=h_{54} h_{50} h_{54}^{-1}=I+799 e_{31}-10241 e_{32}-931 e_{34}+2261 e_{35}, \\
& h_{58}:=h_{55} h_{50} h_{55}^{-1}=I+2983 e_{31}-28755 e_{32}+5325 e_{34}-8094 e_{35} .
\end{aligned}
$$

In particular, $h_{28}, h_{29}, h_{30}, h_{50}, h_{56}, h_{57}$, and $h_{58}$ commute pairwise. An easy calculation shows that

$$
\begin{aligned}
& h_{59}:=h_{56} h_{57}^{2} h_{28}^{-24533} h_{29}^{-20902} h_{30}^{-88456} h_{50}^{55381}=t_{32}(1), \\
& h_{60}:=h_{58}^{-1} h_{57}^{-2} h_{28}^{-893} h_{29}^{866} h_{50}^{4581} h_{59}^{-49237}=t_{34}(1), \\
& h_{61}:=h_{57} h_{28}^{-565} h_{50}^{-799} h_{59}^{10241} h_{60}^{931}=t_{35}(1) .
\end{aligned}
$$

By (43) and Lemma 5.1, we have

$$
t_{12}(1), t_{42}(1), t_{52}(1) \in\langle x, y\rangle \text {. }
$$


Finally, set

$$
\begin{aligned}
& h_{62}:=y x y^{2} x(y x)^{2} y^{2} x y h_{60} y^{2} x y\left(x y^{2}\right)^{2} x y x y^{2}, \\
& h_{63}:=(x y)^{2} x y^{2}(x y)^{5} x y^{2} h_{60} y x\left(y^{2} x\right)^{5} y x\left(y^{2} x\right)^{2}, \\
& h_{64}:=x h_{61} x, \\
& h_{65}:=y x y h_{61} y^{2} x y^{2}, \\
& h_{66}:=y^{2} x y h_{61} y^{2} x y, \\
& h_{67}:=h_{62} h_{51} h_{62}^{-1}=I-48 e_{21}-1617 e_{23}-980 e_{24}-1813 e_{25}, \\
& h_{68}:=h_{63} h_{51} h_{63}^{-1}=I+155 e_{21}-2772 e_{23}-1771 e_{24}+77 e_{25}, \\
& h_{69}:=h_{64} h_{51} h_{64}^{-1}=I+e_{21}+8 e_{23}+8 e_{25}, \\
& h_{70}:=h_{65} h_{51} h_{65}^{-1}=I+e_{21}+40 e_{23}+20 e_{24}+60 e_{25}, \\
& h_{71}:=h_{66} h_{51} h_{66}^{-1}=I+e_{21}-5 e_{23}-5 e_{24}+5 e_{25} .
\end{aligned}
$$

In particular, the elements $h_{51}, h_{67}, \ldots, h_{71}$ commute pairwise, and

$$
h_{72}:=h_{51}^{2164} h_{67}^{26} h_{68}^{5} h_{69}^{216} h_{70}^{992} h_{71}^{-2899}=t_{23}(1) .
$$

Relations (47), (42), (44), and (45) imply that $t_{23}(1), t_{31}(1), t_{34}(1)$, and $t_{35}(1)$ belong to $\langle x, y\rangle$. Using the commutator identity (1), we obtain

$$
t_{21}(1), t_{24}(1), t_{25}(1) \in\langle x, y\rangle .
$$

Using this and also (43), (46), (47), and again the commutator identity (11), we conclude that $\langle x, y\rangle$ contains all the transvections $t_{i j}(1), 1 \leq i \neq j \leq 5$. Therefore, $\langle x, y\rangle$ contains $\mathrm{SL}(5, \mathbb{Z})$. Since $\langle x, y\rangle$ also contains matrices of determinant -1 (e.g., $x)$, the group $\langle x, y\rangle$ coincides with $\mathrm{GL}(5, \mathbb{Z})$. Lemma 2.1 implies that $\langle-x, y\rangle=\mathrm{SL}(5, \mathbb{Z})$.

\section{§. $n=5$ : THE THIRD GENERATING SET}

In this section we consider the case where $X$ and $Y$ are given by (2) with $a_{1}=1$, $a_{2}=-1, a_{3}=1$, and $a_{4}=-3$. Again, it is more convenient to work with a pair of conjugates of $X$ and $Y$. Let

$$
Q=\left(\begin{array}{rrrrr}
1 & 0 & 0 & 2 & 4 \\
1 & 0 & 1 & 3 & 4 \\
1 & 0 & 1 & 2 & 3 \\
0 & 1 & 0 & 0 & -2 \\
0 & 0 & 0 & 0 & 1
\end{array}\right)
$$

and let

$$
\begin{aligned}
& x=Q X Q^{-1}=\left(\begin{array}{rrrrr}
-3 & 0 & 4 & 0 & 4 \\
-1 & -1 & 4 & 0 & 0 \\
-1 & 0 & 2 & 0 & 1 \\
2 & 0 & -2 & -1 & -6 \\
-1 & 0 & 1 & 0 & 2
\end{array}\right), \\
& y=Q Y Q^{-1}=\left(\begin{array}{rrrrr}
2 & -2 & 0 & 1 & 1 \\
3 & -2 & -1 & 1 & -2 \\
2 & -1 & -1 & 1 & 0 \\
-1 & 2 & -2 & -1 & -3 \\
0 & 0 & 0 & 0 & 1
\end{array}\right) .
\end{aligned}
$$

Theorem 6.1. Let $x$ and $y$ be defined by (48) and (49). Then $\langle x, y\rangle=\operatorname{GL}(5, \mathbb{Z})$ and $\langle-x, y\rangle=\mathrm{SL}(5, \mathbb{Z})$. 
Proof. First, we find suitable transvections of level 2. We start with the following matrices:

$$
\begin{aligned}
& h_{1}:=(y x)^{3}\left(y^{2} x\right)^{3} y x y^{2} x, \\
& h_{2}:=h_{1}^{-4}=t_{52}(2), \\
& h_{3}:=y x y^{2} x y x y^{2}, \\
& h_{4}:=y x y x y x y^{2} x y x y x y^{2}, \\
& h_{5}:=\left(x y^{2}\right)^{2} x y x y^{2}(x y)^{2}\left(x y^{2}\right)^{2} x y x y^{2} x y x y^{2} x, \\
& h_{6}:=h_{3} h_{2} h_{3}^{-1}=I+8 e_{12}+2 e_{32}-12 e_{42}+4 e_{52}, \\
& h_{7}:=h_{4}^{2} h_{2} h_{4}^{-2}=I-4 e_{12}-2 e_{32}+8 e_{42}, \\
& h_{8}:=h_{4}^{-2} h_{2} h_{4}^{2}=I-4 e_{12}-2 e_{32}+6 e_{42}, \\
& h_{9}:=h_{5} h_{2} h_{5}^{-1}=I+10 e_{12}+2 e_{32}-14 e_{42}+6 e_{52} .
\end{aligned}
$$

It is easy to check that $h_{2}, h_{6}, \ldots, h_{9}$ commute pairwise and generate the same subgroup as $t_{i 2}(2)$ with $i=1,3,4,5$. More precisely,

$$
\begin{aligned}
& h_{10}:=h_{2}^{-1} h_{6}^{-1} h_{7} h_{8}^{-1} h_{9}=t_{12}(2), \\
& h_{11}:=h_{2}^{2} h_{6}^{-1} h_{8}^{-2}=t_{32}(2), \\
& h_{12}:=h_{7} h_{8}^{-1}=t_{42}(2) .
\end{aligned}
$$

Next, consider

$$
\begin{aligned}
& h_{13}:=(y x)^{3} y^{2} x y, \\
& h_{14}:=(y x)^{6}\left(y^{2} x\right)^{2} y(y x)^{-6}, \\
& h_{15}:=(y x)^{2} y^{2} x y x y, \\
& h_{16}:=h_{13} h_{2} h_{13}^{-1}=I+6 e_{51}-4 e_{52}+4 e_{53}+4 e_{54}, \\
& h_{17}:=h_{13}^{2} h_{2} h_{13}^{-2}=I+4 e_{51}-2 e_{52}+8 e_{53}+4 e_{54}, \\
& h_{18}:=h_{14} h_{2} h_{14}^{-1}=I+16 e_{51}-12 e_{52}+20 e_{53}+14 e_{54}, \\
& h_{19}:=h_{15}^{2} h_{2} h_{15}^{-2}=I-12 e_{51}+8 e_{52}-6 e_{53}-8 e_{54} .
\end{aligned}
$$

The matrices $h_{2}, h_{16}, \ldots, h_{19}$ allow us to find transvections $t_{5 i}(2), i=1,3$. Namely,

$$
\begin{aligned}
& h_{20}:=h_{2}^{-2} h_{16}^{-1} h_{17}^{4} h_{18}^{-2} h_{19}^{-2}=t_{51}(2), \\
& h_{21}:=h_{16}^{2} h_{19}=t_{53}(2) .
\end{aligned}
$$

There is a similar expression for $t_{54}(2)$, but we omit it because it is not used in what follows. Now, we set

$$
\begin{aligned}
& h_{22}:=x y x y^{2} x y x y^{2}(x y)^{3} x, \\
& h_{23}:=\left(y^{2} x\right)^{2}(y x)^{4} y \\
& h_{24}:=h_{22}^{-2} h_{20} h_{22}^{2}=I-6 e_{21}-2 e_{31}-4 e_{41}+4 e_{51}, \\
& h_{25}:=h_{23}^{4} h_{20} h_{23}^{-4}=I+4 e_{21}+2 e_{31}+2 e_{41}+2 e_{51}, \\
& h_{26}:=h_{23}^{-4} h_{20} h_{23}^{4}=I+2 e_{21}+2 e_{51} .
\end{aligned}
$$


The matrices $h_{20}, h_{24}, h_{25}$, and $h_{26}$ commute pairwise and generate the same subgroup as $t_{i 1}(2)$ with $i=2,3,4,5$. Namely,

$$
\begin{aligned}
& h_{27}:=h_{20}^{-1} h_{26}=t_{21}(2), \\
& h_{28}:=h_{20}^{-3} h_{24} h_{25}^{2} h_{26}^{-1}=t_{31}(2), \\
& h_{29}:=h_{20}^{4} h_{24}^{-1} h_{25}^{-1} h_{26}^{-1}=t_{41}(2) .
\end{aligned}
$$

We put

$$
\begin{aligned}
h_{30} & :=y x y, \\
h_{31} & :=h_{30} h_{21} h_{30}^{-1}, \\
h_{32} & :=h_{30}^{-1} h_{21} h_{30}, \\
h_{33} & :=h_{30}^{2} h_{21} h_{30}^{-2}, \\
h_{34} & :=h_{21}^{-6} h_{31}^{5} h_{32}^{3} h_{33}^{-4}=t_{13}(2), \\
h_{35} & :=h_{21}^{2} h_{31}^{-2} h_{32}^{-1} h_{33}=t_{23}(2), \\
h_{36} & :=h_{21}^{-3} h_{31}^{3} h_{32}^{2} h_{33}^{-2}=t_{43}(2)
\end{aligned}
$$

and consider

$$
\begin{aligned}
& h_{37}:=y x\left(y^{2} x\right)^{2}(y x)^{2} y^{2}, \\
& h_{38}:=h_{29} h_{37} h_{36}^{-1} h_{37} h_{36}^{-2}=t_{45}(2), \\
& h_{39}:=(x y)^{3}\left(x y^{2}\right)^{3} x \\
& h_{40}:=(y x)^{3}\left(y^{2} x\right)^{2} y^{2}, \\
& h_{41}:=h_{27} h_{35} h_{11} h_{35}^{-1} h_{10}^{-1} h_{39} h_{10}, \\
& h_{42}:=h_{41} h_{40} h_{1}^{2} h_{34}^{5} h_{40}^{-1} h_{41}^{-1} \\
& h_{43}:=\left(h_{41} h_{40}\right)^{2} h_{1}^{2} h_{34}^{5}\left(h_{41} h_{40}\right)^{-2}, \\
& h_{44}:=h_{42} h_{43}, \\
& h_{45}:=h_{28}^{-1} h_{44} h_{38} h_{2}^{-6} h_{20}^{3} h_{21}^{5} h_{29}^{-20} h_{12}^{37} h_{36}^{-26}=I+e_{43}+e_{51}+e_{52} .
\end{aligned}
$$

Let

$$
\begin{aligned}
& h_{46}:=\left(y x y^{2} x\right)^{2} y^{2} x y x\left(y^{2} x\right)^{2} y^{2}, \\
& h_{47}:=h_{46} h_{45} h_{46}^{-1}, \\
& h_{48}:=h_{41} h_{46} h_{45} h_{46}^{-1} h_{45}^{-1} h_{41}^{-1}, \\
& h_{49}:=h_{46} h_{47} h_{48} h_{46}^{-1}, \\
& h_{50}:=h_{41} h_{48} h_{49} h_{41}^{-1} .
\end{aligned}
$$

Now we are ready to find transvections of level 1:

$$
\begin{aligned}
& h_{51}:=h_{47} h_{48} h_{49} h_{50} h_{2}^{6} h_{20}^{-1} h_{21}^{-13} h_{29}^{-15} h_{12}^{15} h_{36}^{-8}=t_{53}(1), \\
& h_{52}:=h_{45} h_{48} h_{49} h_{50} h_{2}^{6} h_{12}^{15} h_{20}^{-2} h_{21}^{-13} h_{29}^{-15} h_{36}^{-7}=t_{41}(1) .
\end{aligned}
$$

Next, we set

$$
\begin{aligned}
& h_{53}:=h_{13} h_{51} h_{13}^{-1}=I+e_{51}-e_{52}+2 e_{53}+e_{54}, \\
& h_{54}:=h_{13}^{2} h_{51} h_{13}^{-2}=I+e_{51}+3 e_{53}+e_{54}, \\
& h_{55}:=h_{13}^{-1} h_{51} h_{13}=I-2 e_{51}+e_{52}+e_{53}-e_{54}
\end{aligned}
$$


and find more transvections:

$$
\begin{aligned}
& h_{56}:=h_{51}^{3} h_{53}^{-1} h_{55}^{-1}=t_{51}(1), \\
& h_{57}:=h_{51}^{-1} h_{53}^{-1} h_{54}=t_{52}(1), \\
& h_{58}:=h_{51}^{-6} h_{53} h_{54} h_{55}=t_{54}(1), \\
& h_{59}:=h_{37} h_{52} h_{37}=I+2 e_{41}-3 e_{43}-3 e_{45}, \\
& h_{60}:=h_{38}^{2} h_{36}^{-18} h_{12}^{16} h_{29}^{-13} h_{56}^{-7} h_{21}^{-7} h_{2}^{5} h_{47} h_{50} h_{59}=t_{45}(1), \\
& h_{61}:=y x\left(y^{2} x\right)^{3} y x, \\
& h_{62}:=x y^{2}(x y)^{3} x y^{2} x y\left(x y^{2}\right)^{2}(x y)^{2}, \\
& h_{63}:=y^{2} h_{60} y=I-e_{15}-e_{25}-e_{35}, \\
& h_{64}:=y h_{61}^{2} h_{60} h_{61}^{-2} y^{2}=I+4 e_{25}+4 e_{35}+3 e_{45}, \\
& h_{65}:=h_{62}^{2} h_{60} h_{62}^{-2}=I+6 e_{15}+11 e_{25}+4 e_{35}-3 e_{45}, \\
& h_{66}:=h_{62}^{-2} y h_{60} y^{2} h_{62}^{2}=I-7 e_{15}-12 e_{25}-4 e_{35}+5 e_{45}, \\
& h_{67}:=h_{60}^{8} h_{64} h_{65}^{-8} h_{66}^{-7}=t_{15}(1), \\
& h_{68}:=h_{60}^{-6} h_{64}^{-1} h_{65}^{7} h_{66}^{6}=t_{25}(1), \\
& h_{69}:=h_{60}^{-2} h_{63}^{-1} h_{65} h_{66}=t_{35}(1) .
\end{aligned}
$$

Relations (50)-(57) imply that the transvections $t_{5 i}(1), t_{i 5}(1), i=1,2,3,4$, are in $\langle x, y\rangle$. Using the commutator identity (11), we conclude that $\langle x, y\rangle$ contains all the transvections $t_{i j}(1), 1 \leq i \neq j \leq 5$. Therefore, $\langle x, y\rangle$ contains $\operatorname{SL}(5, \mathbb{Z})$. Since $\langle x, y\rangle$ also contains matrices of determinant -1 (e.g., $x$ ), the group $\langle x, y\rangle$ coincides with $\mathrm{GL}(5, \mathbb{Z})$. Lemma2.1 implies that $\langle-x, y\rangle=\mathrm{SL}(5, \mathbb{Z})$.

Proof of Theorem 1.2. This follows immediately from Theorem 3.1, Remark 3.1, and Theorems 3.2, 3.3, 4.1, 5.1, 6.1.

Remark 6.1. It is easy to check that all six pairs of matrices listed in Theorem 1.2 are pairwise nonconjugate.

\section{$\S 7$. The GROUP $\mathrm{SL}(6, \mathbb{Z})$}

As was shown in [12], the problem on $(2,3)$-generation of $\mathrm{SL}(6, \mathbb{Z})$ and the classification of the corresponding generators can be reduced to the analysis of eight cases. Using methods similar to those employed in the proof of Theorems 3.2, 3.3, one can exclude four cases. The answer in the fifth case is positive (see Theorem 7.1 below). However, the author was unable to settle the remaining three cases. For this reason, in the present paper we restrict ourselves to the positive result only.

Consider the matrices

$$
x=\left(\begin{array}{rrrrrr}
1 & 0 & 0 & 0 & 0 & 0 \\
0 & 1 & 0 & 0 & 0 & 0 \\
0 & 0 & -1 & 5 & 1 & -5 \\
1 & 2 & 0 & 1 & 0 & -1 \\
5 & 10 & 0 & 0 & 1 & -5 \\
2 & 4 & 0 & 0 & 0 & -1
\end{array}\right),
$$




$$
y=\left(\begin{array}{rrrrrr}
-1 & 1 & 2 & 2 & 0 & -2 \\
1 & 0 & -1 & -1 & 0 & 1 \\
1 & 3 & 1 & -1 & 0 & 0 \\
1 & 2 & 2 & -5 & -1 & 4 \\
5 & 7 & 1 & -10 & 0 & 5 \\
2 & 3 & 2 & -7 & -1 & 5
\end{array}\right) .
$$

It can be shown that the pair $x, y$ is conjugate to the pair $X, Y$ in [12, Theorem 1] with $a_{1}=3, a_{2}=1, a_{3}=-1, a_{4}=1, b_{1}=0, b_{2}=2, b_{3}=-2$, and $b_{4}=-3$. The choice of $x, y$ instead of $X, Y$ is for technical convenience and simplification of the proof only.

Theorem 7.1. Let $x$ and $y$ be defined by (58) and (59). Then $\langle x, y\rangle=\operatorname{SL}(6, \mathbb{Z})$.

Lemma 7.1. Let $G$ be a subgroup of $\mathrm{SL}(6, \mathbb{Z})$ containing $x, y$, and $t_{36}(a)$ for some integer $a$. Then $G$ also contains

(i) $t_{3 j}(a), j \in\{1,2,4,5\}$;

(ii) $t_{i 6}(a), i \in\{1,2,4,5\}$;

(iii) $t_{4 i}(a), i \in\{1,2,3\}$;

(iv) $t_{63}(a)$.

Proof. As before, by $h$ with subscripts we denote elements of the group $G$ that do not depend on $a$, while by $z_{i}(i=1,2, \ldots)$ we denote elements that do depend on $a$. We start with the matrix

$$
z_{1}=t_{36}(a)
$$

and set

$$
\begin{aligned}
& h_{1}:=y x y^{2} x(y x)^{2}\left(y^{2} x\right)^{2}(y x)^{4} y^{2}, \\
& h_{2}:=(y x)^{5} y^{2} x y^{2}, \\
& z_{2}:=h_{1} z_{1} h_{1}^{-1}=I+a\left(e_{31}+3 e_{32}+3 e_{34}-2 e_{36}\right), \\
& z_{3}:=h_{1}^{-1} z_{1} h_{1}=I+a\left(-8 e_{31}-11 e_{32}-4 e_{34}-2 e_{35}+7 e_{36}\right), \\
& z_{4}:=h_{1}^{2} z_{1} h_{1}^{-2}=I+a\left(17 e_{31}+28 e_{32}+8 e_{34}+3 e_{35}-10 e_{36}\right), \\
& z_{5}:=h_{1}^{-2} z_{1} h_{1}^{2}=I+a\left(9 e_{31}+16 e_{32}-8 e_{34}-3 e_{35}+12 e_{36}\right), \\
& z_{6}:=h_{2}^{4} z_{1} h_{2}^{-4}=I+a\left(e_{31}+e_{32}-2 e_{34}\right) .
\end{aligned}
$$

In particular, the matrices $z_{1}, \ldots, z_{6}$ commute pairwise. It is easy to check that

$$
\begin{aligned}
z_{7} & :=z_{1}^{101} z_{2}^{46} z_{3}^{-9} z_{4}^{-9} z_{5}^{-3} z_{6}^{63}=t_{31}(a), \\
z_{8} & :=z_{1}^{123} z_{2}^{56} z_{3}^{-9} z_{4}^{-10} z_{5}^{-4} z_{6}^{78}=t_{32}(a), \\
z_{9} & :=z_{1}^{-156} z_{2}^{-71} z_{3}^{12} z_{4}^{13} z_{5}^{5} z_{6}^{-99}=t_{34}(a), \\
z_{10} & :=z_{1}^{-229} z_{2}^{-106} z_{3}^{19} z_{4}^{20} z_{5}^{7} z_{6}^{-145}=t_{35}(a),
\end{aligned}
$$

which proves (i). Now we set

$$
\begin{aligned}
h_{3} & :=\left(y x y^{2} x\right)^{2} x, \\
h_{4} & :=\left(y^{2} x y x\right)^{2} x, \\
h_{5} & :=y^{2} x y x\left(y^{2} x\right)^{3}(y x)^{3} y^{2} x y, \\
h_{6} & :=(y x)^{2} y^{2} x y x\left(y^{2} x\right)^{2}(y x)^{2} y^{2} x(y x)^{3} y^{2}, \\
z_{11} & :=h_{3} z_{1} h_{3}^{-1}=I+a\left(2 e_{36}+3 e_{56}\right), \\
z_{12} & :=h_{4} z_{1} h_{4}^{-1}=I+a\left(-e_{16}+e_{26}-e_{56}\right), \\
z_{13} & :=h_{5} z_{1} h_{5}^{-1}=I+a\left(-2 e_{16}+e_{26}+e_{36}+e_{46}-5 e_{56}\right),
\end{aligned}
$$




$$
\begin{aligned}
& z_{14}:=h_{6} z_{1} h_{6}^{-1}=I+a\left(7 e_{16}-5 e_{26}+9 e_{36}-e_{46}+16 e_{56}\right), \\
& z_{15}:=h_{6}^{-1} z_{12} h_{6}=I+a\left(4 e_{16}-2 e_{26}+e_{36}-3 e_{56}\right) .
\end{aligned}
$$

The matrices $z_{1}, z_{11}, z_{12}, z_{13}, z_{14}$, and $z_{15}$ commute pairwise. A direct calculation shows that

$$
\begin{aligned}
& z_{16}:=z_{1} z_{11}^{4} z_{12}^{-2} z_{13}^{-1} z_{14}^{-1} z_{15}=t_{16}(a), \\
& z_{17}:=z_{1}^{-6} z_{11}^{-2} z_{12}^{5} z_{13} z_{14}=t_{26}(a), \\
& z_{18}:=z_{1}^{-9} z_{11}^{-1} z_{12}^{3} z_{13}^{2} z_{14}=t_{46}(a), \\
& z_{19}:=z_{1}^{-7} z_{11}^{-6} z_{12}^{6} z_{13}^{2} z_{14}^{2} z_{15}^{-1}=t_{56}(a) .
\end{aligned}
$$

This proves (ii). Next, we set

$$
\begin{aligned}
h_{7} & :=y x\left(y^{2} x\right)^{5}(y x)^{2} y^{2}, \\
z_{20} & :=z_{1}^{5} z_{7}^{-10} z_{8}^{-20} z_{18} x z_{18} x h_{7}^{4} z_{18} h_{7}^{-4}=t_{42}(a), \\
h_{8} & :=\left(x y x y^{2}\right)^{2}(x y)^{2}\left(x y^{2}\right)^{2}(x y)^{4} x y^{2}, \\
h_{9} & :=h_{8}^{2} x h_{8}^{-2} x \\
z_{21} & :=z_{1}^{-125} z_{7}^{81} z_{8}^{500} h_{9} z_{7} h_{9}^{-1} x z_{18}^{-25} x z_{18}^{-25} z_{20}^{100}=t_{41}(a), \\
h_{10} & :=\left(x y\left(x y^{2}\right)^{2}(x y)^{2}\left(x y^{2}\right)^{2} x y\right)^{2}, \\
h_{11} & :=\left(y x y^{2} x y^{2} x y x\right)^{4}, \\
z_{22} & :=h_{11} z_{19} h_{11}^{-1} z_{19}^{-1}=t_{54}(3 a), \\
z_{23} & :=z_{1}^{12} z_{7}^{-18} z_{8}^{-36} z_{9}^{-18} h_{10}^{-1} z_{19}^{-1} h_{10} z_{19}^{10} z_{22}^{-5}=I+a\left(15 e_{51}+30 e_{52}\right), \\
z_{24} & :=h_{10} z_{20}^{2} z_{21} h_{10}^{-1} \\
& =I+a\left(30 e_{31}+60 e_{32}+4 e_{41}+8 e_{42}+30 e_{51}+60 e_{52}+3 e_{61}+6 e_{62}\right), \\
z_{25} & :=z_{7}^{-30} z_{8}^{-60} z_{20}^{-10} z_{21}^{-5} z_{23}^{-1} z_{24} \\
& =I+a\left(-e_{41}-2 e_{42}+15 e_{51}+30 e_{52}+3 e_{61}+6 e_{62}\right), \\
h_{12} & :=x y^{2} x y x \\
z_{26} & :=h_{12} z_{25} h_{12}^{-1}=t_{43}(a) .
\end{aligned}
$$

Relations (60), (61), (62) prove (iii). Finally, let

$$
\begin{aligned}
z_{27} & :=h_{11} z_{20}^{-6} z_{21}^{-3} h_{11}^{-1} z_{23}^{-2} z_{20}^{16} z_{21}^{8} \\
& =I+a\left(5 e_{41}+10 e_{42}+15 e_{51}+30 e_{52}+9 e_{61}+18 e_{62}\right), \\
h_{13} & :=\left(y^{2} x y x\right)^{3}, \\
z_{28} & :=h_{13}^{-2} z_{27} h_{13}^{2}=I+a\left(e_{61}+2 e_{62}\right), \\
z_{29} & :=h_{12} z_{28}^{-4} z_{23}^{-1} h_{12}=t_{63}(a) .
\end{aligned}
$$

The last-written relation completes the proof of the lemma. 
Proof of Theorem 7.1. We keep the notation introduced in the proof of Lemma 7.1, Set

$$
\begin{aligned}
& h_{14}:=\left(y^{2} x y x y^{2} x y^{2} x y^{2}\right)^{4}, \\
& h_{15}:=\left(y x y^{2} x\right)^{4}, \\
& h_{16}:=h_{14} h_{15} h_{14}^{-1} h_{15}^{-1}, \\
& h_{17}:=x y x y^{2} x, \\
& h_{18}:=h_{17} h_{16}^{-1} h_{17}^{-1} x h_{16} x=t_{36}(3) .
\end{aligned}
$$

Lemma 7.1 applied to $a=3$ shows that the matrices

$$
\begin{aligned}
& h_{19}:=t_{31}(3), \quad h_{20}:=t_{32}(3), \quad h_{21}:=t_{34}(3), \quad h_{22}:=t_{35}(3), \\
& h_{23}:=t_{41}(3), \quad h_{24}:=t_{42}(3), \quad h_{25}:=t_{43}(3), \quad h_{26}:=t_{46}(3)
\end{aligned}
$$

are in $\langle x, y\rangle$. Now, we consider

$$
\begin{aligned}
& h_{27}:=h_{21}^{-2} x h_{25}^{-1} x h_{21}^{2}, \\
& h_{28}:=h_{7}^{4} x h_{9} x h_{7}^{-4}, \\
& h_{29}:=h_{27} h_{21}^{-1} h_{28} h_{21} h_{25} h_{21}^{2}, \\
& h_{30}:=h_{18}^{-15} h_{19}^{14} h_{20}^{22} h_{22}^{4} h_{26}^{-1} h_{24}^{2} h_{23} h_{29}=t_{32}(1), \\
& h_{31}:=x y^{2}(x y)^{2} x y^{2}(x y)^{3} x, \\
& h_{32}:=h_{1}^{-1} h_{30} h_{1}=I+9 e_{31}+15 e_{32}-2 e_{34}-e_{35}+3 e_{36}, \\
& h_{33}:=h_{2}^{-4} h_{30} h_{2}^{4}=I+e_{31}+2 e_{32}-2 e_{34}+2 e_{36}, \\
& h_{34}:=h_{31}^{2} h_{30} h_{31}^{-2}=I+2 e_{31}+4 e_{32}-3 e_{34}+e_{36}, \\
& h_{35}:=h_{1}^{2} h_{30} h_{1}^{-2}=I-24 e_{31}-37 e_{32}-9 e_{34}-5 e_{35}+14 e_{36} .
\end{aligned}
$$

In particular, $h_{18}, h_{30}, h_{32}, h_{33}, h_{34}$, and $h_{35}$ commute pairwise. An easy calculation shows that

$$
h_{36}:=h_{18}^{-91} h_{30}^{26} h_{32}^{5} h_{33}^{205} h_{34}^{-137} h_{35}^{-1}=t_{36}(1) .
$$

In particular,

$$
t_{36}(1) \in\langle x, y\rangle \text {. }
$$

Applying Lemma 7.1 with $a=1$, we conclude that

$$
\begin{aligned}
& \left\{t_{31}(1), t_{32}(1), t_{34}(1), t_{35}(1)\right\} \subset\langle x, y\rangle, \\
& \left\{t_{16}(1), t_{26}(1), t_{46}(1), t_{56}(1)\right\} \subset\langle x, y\rangle, \\
& t_{63}(1) \in\langle x, y\rangle .
\end{aligned}
$$

Relations (64), (66) and the commutator identity (10) imply that

$$
\left\{t_{61}(1), t_{62}(1), t_{64}(1), t_{65}(1)\right\} \subset\langle x, y\rangle .
$$

Applying (63), (65), (66), (67), and the commutator identity (1), we see that all the transvections $t_{i j}(1)$ with $1 \leq i \neq j \leq 6$ are in $\langle x, y\rangle$. Hence, $\langle x, y\rangle=\mathrm{SL}(6, \mathbb{Z})$.

\section{§8. Yet Another $(2,3)$-Generating SET FOR PSL $(6, \mathbb{Z})$}

Since the group $\mathrm{SL}(6, \mathbb{Z})$ is $(2,3)$-generated, so is the group $\operatorname{PSL}(6, \mathbb{Z})$. On the other hand, it is natural to ask whether $\operatorname{PSL}(6, \mathbb{Z})$ has a pair of $(2,3)$-generators different from those "inherited" from $\mathrm{SL}(6, \mathbb{Z})$. For instance, we may look for generators that are the projective images of matrices satisfying the relations

$$
x^{2}=-I, \quad y^{3}=I \text {. }
$$


The following theorem gives an affirmative answer.

Theorem 8.1. Let

$$
x=\left(\begin{array}{rrrrrr}
0 & -1 & 0 & 0 & 0 & 0 \\
1 & 0 & 0 & 0 & 0 & 0 \\
0 & 0 & 0 & 0 & -1 & 0 \\
0 & 0 & 0 & 0 & -1 & -1 \\
0 & 0 & 1 & 0 & 0 & 0 \\
0 & 0 & -1 & 1 & 0 & 0
\end{array}\right), \quad y=\left(\begin{array}{rrrrrr}
1 & 0 & 1 & 0 & 0 & 0 \\
0 & -1 & 0 & 0 & -1 & 0 \\
-1 & 0 & -1 & 0 & 0 & 1 \\
0 & 1 & -1 & 1 & 1 & 0 \\
0 & 1 & 0 & 0 & 0 & 0 \\
1 & 0 & 0 & 0 & 0 & 0
\end{array}\right) .
$$

Then $x^{2}=-I, y^{3}=I$, and $\langle x, y\rangle=\mathrm{SL}(6, \mathbb{Z})$. In particular, the projective images of $x$ and $y$ are of order 2 and 3 , respectively, and generate the group $\operatorname{PSL}(6, \mathbb{Z})$.

Remark 8.1. In contrast to the preceding sections, it is not known whether (up to $\mathrm{GL}(6, \mathbb{Z})$-conjugation) there are finitely many generating pairs for $\mathrm{SL}(6, \mathbb{Z})$ that satisfy (68). Moreover, it seems plausible that there are infinitely many pairwise nonconjugate generating sets. For this reason, the following method of finding specific generators as in Theorem 8.1 was used. Matrices $x_{0}$ and $y_{0}$ of the form

$$
x_{0}=\left(\begin{array}{rrrrrr}
0 & 0 & -1 & 0 & a_{1} & a_{2} \\
0 & 0 & 0 & -1 & a_{3} & a_{4} \\
1 & 0 & 0 & 0 & a_{2} & -a_{1} \\
0 & 1 & 0 & 0 & a_{4} & -a_{3} \\
0 & 0 & 0 & 0 & 0 & -1 \\
0 & 0 & 0 & 0 & 1 & 0
\end{array}\right), \quad y_{0}=\left(\begin{array}{rrrrrr}
1 & 0 & 0 & 0 & a_{5} & a_{6} \\
0 & 1 & 0 & 0 & a_{7} & a_{8} \\
0 & 0 & 0 & 0 & -1 & 0 \\
0 & 0 & 0 & 0 & 0 & -1 \\
0 & 0 & 1 & 0 & -1 & 0 \\
0 & 0 & 0 & 1 & 0 & -1
\end{array}\right)
$$

were sought so as to satisfy the following natural necessary condition: for any prime $p$ not exceeding some given bound, the matrices $x_{0} \bmod p$ and $y_{0} \bmod p$ generate $\operatorname{SL}(6, p)$. The following set, which passed all tests, was found:

$$
\left(a_{1}, a_{2}, a_{3}, a_{4}, a_{5}, a_{6}, a_{7}, a_{8}\right)=(1,-1,0,1,-1,-1,1,0) .
$$

It turns out that, for this set, the matrices $x_{0}$ and $y_{0}$ generate $\operatorname{SL}(6, \mathbb{Z})$. However, for technical reasons it is more convenient to work not with $x_{0}, y_{0}$, but rather with a conjugate pair $x, y\left(x=Q x_{0} Q^{-1}, y=Q y_{0} Q^{-1}\right.$ for a suitable integral matrix $\left.Q\right)$. Note that the corresponding generators were found by means of a computer search; however, the rigorous proof below is computer-independent.

Proof of Theorem 8.1. Relations (68) are trivial.

Consider the following matrices:

$$
\begin{aligned}
& h_{1}:=x y x^{-1} y^{-1}, \\
& h_{2}:=\left(y^{2} x^{-1} y x\right)^{2}, \\
& h_{3}:=\left(h_{2} h_{1}\right)^{2} h_{2}^{-1}\left(h_{2} h_{1}\right)^{-2} h_{2}, \\
& h_{4}:=\left(h_{2} h_{1}\right)^{4} h_{2}^{-1}\left(h_{2} h_{1}\right)^{-4} h_{2}, \\
& h_{5}:=h_{1} h_{2} h_{1} h_{2}^{-1} h_{1}^{-1} h_{2}^{-1} h_{1}^{-1} h_{2}, \\
& h_{6}:=h_{3}^{-9} h_{4} h_{5}^{3} .
\end{aligned}
$$

In particular, they are all of block shape

$$
\left(\begin{array}{ll}
* & 0 \\
* & *
\end{array}\right)
$$

where the upper left-hand block is of size $2 \times 2$, and the lower right-hand block is of size $4 \times 4$. Moreover, $h_{6}$ has the form

$$
I+k_{31} e_{31}+k_{32} e_{32}+k_{41} e_{41}+k_{42} e_{42}+k_{51} e_{51}+k_{52} e_{52}+k_{61} e_{61}+k_{62} e_{62} .
$$


TABLE 2

\begin{tabular}{|r|r|r|r|r|r|r|r|r|}
\hline & $k_{31}$ & $k_{32}$ & $k_{41}$ & $k_{42}$ & $k_{51}$ & $k_{52}$ & $k_{61}$ & $k_{62}$ \\
\hline$h_{6}$ & 3 & 5 & 1 & -3 & 2 & 3 & -2 & 5 \\
$h_{7}$ & 3 & 5 & 9 & 10 & 2 & 3 & 3 & 13 \\
$h_{8}$ & 3 & 5 & 10 & 12 & 2 & 3 & 4 & 14 \\
$h_{9}$ & 3 & 5 & 7 & 7 & 2 & 3 & 2 & 11 \\
$h_{10}$ & 2 & -5 & 4 & -2 & 1 & -3 & -4 & -2 \\
$h_{11}$ & 0 & -8 & 2 & 3 & -2 & -8 & -3 & -5 \\
$h_{12}$ & 1 & 2 & 15 & -9 & 1 & 1 & -24 & 17 \\
$h_{13}$ & -3 & -13 & -1 & -10 & 9 & 10 & -12 & -15 \\
$h_{14}$ & -5 & -16 & 2 & 3 & 6 & 5 & -3 & -5 \\
$h_{15}$ & -6 & -17 & 2 & 3 & 7 & 7 & -3 & -5 \\
\hline
\end{tabular}

Now we consider matrices of the same form (69). (For each matrix, the values of the coefficients $k_{i j}$ are listed in Table 2.)

$$
\begin{aligned}
h_{7} & :=h_{3}^{-8} h_{4} h_{3}^{-1} h_{5}^{3}, \\
h_{8} & :=h_{3}^{-9} h_{5}^{3} h_{4}, \\
h_{9} & :=h_{3}^{-9} h_{5}^{2} h_{4} h_{5}, \\
h_{10} & :=h_{1} h_{6} h_{1}^{-1}, \\
h_{11} & :=h_{1}^{-1} h_{6} h_{1}, \\
h_{12} & :=h_{2}^{-1} h_{6} h_{2}, \\
h_{13} & :=h_{1} h_{7} h_{1}^{-1}, \\
h_{14} & :=h_{1}^{-1} h_{7} h_{1}, \\
h_{15} & :=h_{1}^{-1} h_{8} h_{1} .
\end{aligned}
$$

Clearly, the matrices $h_{7}, \ldots, h_{15}$ commute pairwise. We show that, in fact, the subgroup generated by them coincides with

$$
\left\langle t_{31}(1), t_{41}(1), t_{51}(1), t_{61}(1), t_{32}(1), t_{42}(1), t_{52}(1), t_{62}(1)\right\rangle .
$$

Indeed, we have

$$
\begin{aligned}
& h_{16}:=h_{7}^{1034} h_{8}^{-808} h_{9}^{-149} h_{10}^{7} h_{11}^{121} h_{12}^{-21} h_{13}^{-8} h_{14}^{-685} h_{15}^{612}=t_{31}(1), \\
& h_{17}:=h_{7}^{-2087} h_{8}^{1686} h_{9}^{221} h_{10}^{-17} h_{11}^{-286} h_{12}^{46} h_{13}^{19} h_{14}^{1623} h_{15}^{-1450}=t_{41}(1), \\
& h_{18}:=h_{7}^{-4604} h_{8}^{3681} h_{9}^{543} h_{10}^{-36} h_{11}^{-603} h_{12}^{99} h_{13}^{40} h_{14}^{3423} h_{15}^{-3058}=t_{51}(1), \\
& h_{19}:=h_{7}^{-4701} h_{8}^{3803} h_{9}^{490} h_{10}^{-39} h_{11}^{-649} h_{12}^{104} h_{13}^{43} h_{14}^{365} h_{15}^{-3292}=t_{61}(1), \\
& h_{20}:=h_{7}^{128} h_{8}^{-121} h_{9}^{12} h_{10}^{2} h_{11}^{31} h_{12}^{-4} h_{13}^{-2} h_{14}^{-177} h_{15}^{158}=t_{32}(1), \\
& h_{21}:=h_{7}^{4698} h_{8}^{-3801} h_{9}^{-489} h_{10}^{39} h_{11}^{649} h_{12}^{-104} h_{13}^{-43} h_{14}^{-3685} h_{15}^{3292}=t_{42}(1), \\
& h_{22}:=h_{7}^{-2664} h_{8}^{2186} h_{9}^{233} h_{10}^{-24} h_{11}^{-391} h_{12}^{61} h_{13}^{26} h_{14}^{2222} h_{15}^{-1985}=t_{52}(1), \\
& h_{23}:=h_{7}^{-2609} h_{8}^{2114} h_{9}^{267} h_{10}^{-22} h_{11}^{-363} h_{12}^{58} h_{13}^{24} h_{14}^{2062} h_{15}^{-1842}=t_{62}(1) .
\end{aligned}
$$


Now we use these matrices for finding more transvections in $\langle x, y\rangle$. Consider

$$
\begin{aligned}
& h_{24}:=y h_{17} y^{-1}=t_{46}(1), \\
& h_{25}:=y h_{18}^{-1} h_{17} y^{-1}=t_{26}(1), \\
& h_{26}:=y h_{19} y^{-1}=t_{36}(1), \\
& h_{27}:=y h_{16} h_{17} h_{19} y^{-1}=t_{16}(1), \\
& h_{28}:=y^{-1} h_{16} y h_{19}^{-1}=t_{63}(1), \\
& h_{29}:=y^{-1} h_{20}^{-1} y h_{23}^{-1}=t_{65}(1), \\
& h_{30}:=x h_{24}^{-1} x^{-1} h_{28}=t_{64}(1), \\
& h_{31}:=h_{22} h_{25} h_{22}^{-1} h_{25}^{-1}=t_{56}(1) .
\end{aligned}
$$

In particular, we already know that $t_{6 k}(1)$ and $t_{i 6}(1)$ for all $i, k=1, \ldots, 5$ are in $\langle x, y\rangle$. Using identity (11) we conclude that $t_{i k}(1) \in\langle x, y\rangle$ for all $i, k, i \neq k$. Therefore, $\langle x, y\rangle=$ $\mathrm{SL}(6, \mathbb{Z})$, which proves the theorem.

\section{§9. The GRoup SL $(7, \mathbb{Z})$}

In this section we prove the following theorem.

Theorem 9.1. The groups $\mathrm{SL}(7, \mathbb{Z})$ and $\mathrm{GL}(7, \mathbb{Z})$ are $(2,3)$-generated. More precisely, let

$$
\begin{aligned}
x & =\left(\begin{array}{rrrrrrr}
-1 & 0 & 0 & 0 & 0 & 0 & 0 \\
16 & 1 & -18 & 3 & 0 & -3 & 3 \\
0 & 0 & -1 & 0 & 0 & 0 & 0 \\
-25 & 0 & 25 & 0 & 0 & 1 & -5 \\
-11 & 0 & 12 & 0 & 1 & 0 & -3 \\
-25 & 0 & 25 & 1 & 0 & 0 & -5 \\
0 & 0 & 0 & 0 & 0 & 0 & -1
\end{array}\right) \\
y & =\left(\begin{array}{rrrrrrr}
19 & -4 & -6 & -12 & 18 & 0 & -3 \\
18 & -1 & -12 & -6 & 10 & -1 & 3 \\
20 & -5 & -4 & -15 & 23 & 0 & -6 \\
-2 & 8 & -22 & 25 & -40 & -1 & 21 \\
-12 & 8 & -12 & 24 & -37 & -1 & 16 \\
-12 & 15 & -33 & 45 & -73 & -1 & 40 \\
-7 & 1 & 4 & 3 & -4 & 0 & 0
\end{array}\right) .
\end{aligned}
$$

Then $x^{2}=y^{3}=1$ and $\langle x, y\rangle=\mathrm{SL}(7, \mathbb{Z}),\langle-x, y\rangle=\mathrm{GL}(7, \mathbb{Z})$.

Remark 9.1. The method of finding the matrices $x, y$ is similar to that used in [13] for $\mathrm{GL}(6, \mathbb{Z})$. We looked for matrices $x_{0}, y_{0}$ of the form

$$
x_{0}=\left(\begin{array}{ccccccc}
0 & 0 & 0 & 1 & 0 & 0 & a_{1} \\
0 & 0 & 0 & 0 & 1 & 0 & a_{2} \\
0 & 0 & 0 & 0 & 0 & 1 & a_{3} \\
1 & 0 & 0 & 0 & 0 & 0 & a_{1} \\
0 & 1 & 0 & 0 & 0 & 0 & a_{2} \\
0 & 0 & 1 & 0 & 0 & 0 & a_{3} \\
0 & 0 & 0 & 0 & 0 & 0 & -1
\end{array}\right),
$$




$$
y_{0}=\left(\begin{array}{rrrrrrr}
1 & 0 & 0 & a_{4} & a_{7} & 0 & a_{10} \\
0 & 1 & 0 & a_{5} & a_{8} & 0 & a_{11} \\
0 & 0 & 1 & a_{6} & a_{9} & 0 & a_{12} \\
0 & 0 & 0 & 0 & -1 & 0 & 0 \\
0 & 0 & 0 & 1 & -1 & 0 & 0 \\
0 & 0 & 0 & 0 & 0 & 0 & -1 \\
0 & 0 & 0 & 0 & 0 & 1 & -1
\end{array}\right) .
$$

Obviously, $x_{0}^{2}=y_{0}^{3}=I$. Moreover, we imposed an extra condition on the characteristic polynomial of the commutator of $x_{0}$ and $y_{0}$ :

$$
\chi_{x_{0} y_{0} x_{0}^{-1} y_{0}^{-1}}(\lambda)=(\lambda-1)(\lambda+1)^{2}\left(\lambda^{2}-\lambda+1\right)\left(\lambda^{2}+\lambda+1\right) .
$$

If, in addition, the eigenspace of $x_{0} y_{0} x_{0}^{-1} y_{0}^{-1}$ relative to -1 is one-dimensional, then

$$
\left(x_{0} y_{0} x_{0}^{-1} y_{0}^{-1}\right)^{6}
$$

is a transvection (not necessarily an elementary transvection). Condition (72) can be written as a system of polynomial equations. Next, we looked for "small" solutions of this system that satisfy the following natural necessary condition: for primes $p$ not exceeding some given bound, the matrices $x_{0} \bmod p$ and $y_{0} \bmod p$ generate $\mathrm{SL}(7, p)$. In this way we found the following set of parameters that passed all the tests: $a_{1}=1$, $a_{2}=1, a_{3}=-2, a_{4}=1, a_{5}=0, a_{6}=1, a_{7}=-1, a_{8}=-1, a_{9}=0, a_{10}=1, a_{11}=0$, $a_{12}=-3$. It turns out that, for this set, the matrices $x_{0}$ and $y_{0}$ actually generate $\operatorname{SL}(7, \mathbb{Z})$. It is more convenient to work not with $x_{0}, y_{0}$, but rather with a conjugate pair $x, y$ given by (69), (70); $x=Q x_{0} Q^{-1}, y=Q y_{0} Q^{-1}$ for a suitable integral matrix $Q$. The choice of conjugates was made, in particular, so as to guarantee that $\left(x y x^{-1} y^{-1}\right)^{6}$ is an elementary transvection.

Proof of Theorem 9.1. First, we note that

$$
h_{1}:=\left(x y x y^{2}\right)^{6}=t_{21}(1) \text {. }
$$

Take

$$
h_{2}:=x y x y^{2} x y x y^{2} x y^{2} x y .
$$

Then

$$
h_{3}:=h_{2} h_{1}^{-1} h_{2}^{-1}=t_{12}(1) .
$$

We set

$$
\begin{aligned}
& h_{4}:=x y x y^{2}, \\
& h_{5}:=h_{2} h_{4} h_{2}^{-1},
\end{aligned}
$$

and

$$
\begin{aligned}
Q_{m, j, k} & :=h_{1}^{-k-1} h_{4}^{m} h_{3}^{-1} h_{4}^{-m} h_{1}^{k} h_{3} h_{1} h_{3}^{-1} h_{1}^{-k} h_{4}^{m} h_{3} h_{4}^{-m} h_{1}^{k+1} h_{3}^{j}, \\
R_{m, j, k} & :=h_{1}^{-1} h_{3}^{-k} h_{5}^{m} h_{1}^{-1} h_{5}^{-m} h_{3}^{k} h_{1} h_{3} h_{1}^{-1} h_{3}^{-k} h_{5}^{m} h_{1} h_{5}^{-m} h_{3}^{k} h_{1} h_{3}^{j} .
\end{aligned}
$$

A direct calculation shows that

$$
\begin{aligned}
h_{6} & :=Q_{1,12,-9}=I+121 e_{13}-22 e_{14}+77 e_{15}-11 e_{16}-88 e_{17}, \\
h_{7} & :=Q_{-1,12,-4}=I+44 e_{13}-44 e_{14}+44 e_{15}+11 e_{16}-11 e_{17}, \\
h_{8} & :=Q_{2,-14,-10}=I-255 e_{13}+75 e_{14}-60 e_{15}-45 e_{16}-30 e_{17}, \\
h_{9} & :=Q_{-2,-14,23}=I+285 e_{13}+45 e_{14}-105 e_{15}+30 e_{16}-30 e_{17}, \\
h_{10} & :=Q_{-3,-6,2}=I+14 e_{13}+14 e_{14}-7 e_{16}+14 e_{17}, \\
h_{11} & :=R_{1,-12,-4}=I+253 e_{13}-110 e_{14}+198 e_{15}+11 e_{16}-77 e_{17},
\end{aligned}
$$




$$
\begin{aligned}
h_{12} & :=R_{2,14,23}=I+1155 e_{13}-735 e_{14}+1305 e_{15}+30 e_{16}-480 e_{17}, \\
h_{13} & :=R_{-3,6,3}=I+140 e_{13}-63 e_{14}+140 e_{15}-7 e_{16}-105 e_{17} .
\end{aligned}
$$

In particular, $h_{6}, h_{7}, \ldots, h_{13}$ generate an Abelian subgroup. By standard linear algebra, we can find another basis for the same subgroup:

$$
\begin{aligned}
& h_{14}:=h_{6}^{219} h_{7}^{2} h_{8}^{-149} h_{9}^{-100} h_{10}^{-20} h_{11}^{-52} h_{12}^{-23} h_{13}^{28}=t_{13}(1), \\
& h_{15}:=h_{6}^{476} h_{7}^{460} h_{8}^{-246} h_{9}^{-308} h_{10}^{109} h_{11}^{456} h_{12}^{-164} h_{13}^{141}=I+e_{14}+e_{17}, \\
& h_{16}:=h_{6}^{200} h_{7}^{244} h_{8}^{-105} h_{9}^{-143} h_{10}^{71} h_{11}^{240} h_{12}^{-82} h_{13}^{86}=t_{15}(1), \\
& h_{17}:=h_{6}^{-370} h_{7}^{-516} h_{8}^{398} h_{9}^{350} h_{10}^{-325} h_{11}^{-212} h_{12}^{176} h_{13}^{-542}=t_{16}(1), \\
& h_{18}:=h_{6}^{138} h_{7}^{-87} h_{8}^{161} h_{9}^{46} h_{10}^{-258} h_{11}^{249} h_{12}^{18} h_{13}^{-465}=t_{17}(3) .
\end{aligned}
$$

Remark 9.2. It is not too difficult to show that $h_{14}, \ldots, h_{18}$ is, in fact, a basis of the group generated by $h_{6}, \ldots, h_{13}$. Thus, it is impossible to define $t_{17}(1)$ and $t_{14}(1)$ by using only $h_{6}, \ldots, h_{13}$.

Observe that

$$
h_{5} h_{14}^{-1} h_{5}^{-1}=I+7 e_{12}+e_{13}+7 e_{14}-13 e_{15}+e_{16}+2 e_{17} .
$$

Therefore,

$$
h_{19}:=h_{5} h_{14}^{-1} h_{5}^{-1} h_{3}^{-7} h_{14}^{-1} h_{15}^{-7} h_{16}^{13} h_{17}^{-1} h_{18}^{2}=t_{17}(1) .
$$

Combining this with (76), we conclude that

$$
h_{20}:=h_{15} h_{19}^{-1}=t_{14}(1) .
$$

In a similar way, we define

$$
\begin{aligned}
S_{m, j, k} & :=h_{1}^{j} h_{3}^{-1} h_{1}^{-k} h_{4}^{m} h_{3}^{-1} h_{4}^{-m} h_{1}^{k} h_{3} h_{1} h_{3}^{-1} h_{1}^{-k} h_{4}^{m} h_{3} h_{4}^{-m} h_{1}^{k} h_{3}, \\
T_{m, j, k} & :=h_{1}^{j} h_{3}^{-k-1} h_{5}^{m} h_{1}^{-1} h_{5}^{-m} h_{3}^{k} h_{1} h_{3} h_{1}^{-1} h_{3}^{-k} h_{5}^{m} h_{1} h_{5}^{-m} h_{3}^{k+1}
\end{aligned}
$$

and consider

$$
\begin{aligned}
& h_{21}:=S_{1,10,2}=I-121 e_{31}+913 e_{41}+550 e_{51}+1518 e_{61}-88 e_{71}, \\
& h_{22}:=S_{-1,10,7}=I+33 e_{31}-165 e_{41}-99 e_{51}-451 e_{61}+66 e_{71}, \\
& h_{23}:=S_{2,-16,-25}=I+120 e_{31}-585 e_{41}-435 e_{51}-1230 e_{61}+120 e_{71}, \\
& h_{24}:=S_{-2,-16,8}=I-45 e_{31}+525 e_{41}+345 e_{51}+915 e_{61}-45 e_{71}, \\
& h_{25}:=S_{-3,-8,-5}=I+112 e_{41}+56 e_{51}+133 e_{61}-21 e_{71}, \\
& h_{26}:=T_{-1,-10,2}=I-77 e_{31}+671 e_{41}+396 e_{51}+1221 e_{61}-66 e_{71}, \\
& h_{27}:=T_{2,16,8}=I-675 e_{31}+3990 e_{41}+2550 e_{51}+6945 e_{61}-360 e_{71}, \\
& h_{28}:=T_{-3,8,-4}=I-126 e_{31}+805 e_{41}+497 e_{51}+1484 e_{61}-84 e_{71} .
\end{aligned}
$$

The matrices $h_{21}, \ldots, h_{28}$ generate an Abelian subgroup, and we can find its basis. Set

$$
\begin{aligned}
& h_{29}:=h_{21}^{778} h_{22}^{-448} h_{23}^{92} h_{24}^{143} h_{25}^{-1089} h_{26}^{-106} h_{27}^{-6} h_{28}^{-731}=I+e_{31}+2 e_{71}, \\
& h_{30}:=h_{21}^{1618} h_{22}^{185} h_{23}^{-424} h_{24}^{-412} h_{25}^{-1854} h_{26}^{475} h_{27}^{-194} h_{28}^{-1013}=I+e_{41}+2 e_{71}, \\
& h_{31}:=h_{21}^{-1151} h_{22}^{-1167} h_{23}^{988} h_{24}^{1197} h_{25}^{1131} h_{26}^{-1694} h_{27}^{225} h_{28}^{1143}=I+e_{51}+2 e_{71}, \\
& h_{32}:=h_{21}^{-213} h_{22}^{-155} h_{23}^{99} h_{24}^{76} h_{25}^{148} h_{26}^{33} h_{27}^{67} h_{28}^{-148}=t_{61}(1), \\
& h_{33}:=h_{21}^{624} h_{22}^{-1038} h_{23}^{326} h_{24}^{287} h_{25}^{-1326} h_{26}^{240} h_{27}^{191} h_{28}^{-1833}=t_{71}(3) .
\end{aligned}
$$

Note that

$$
h_{34}:=x h_{32}^{-1} x h_{1}^{3}=t_{41}(1) .
$$


Using (82), (83), (84), (86) and (87), we conclude that

$$
\begin{aligned}
h_{35} & :=h_{34} h_{30}^{-1} h_{33}=t_{71}(1), \\
h_{36} & :=h_{29} h_{35}^{-2}=t_{31}(1), \\
h_{37} & :=h_{31} h_{35}^{-2}=t_{51}(1) .
\end{aligned}
$$

Relations (73)-(75), (77), (78), (80), (81), (85), (87), and (88)-(90) show that the group $\langle x, y\rangle$ contains the transvections $t_{i 1}(1), t_{1 k}(1)$ for all $i, k \in\{2,3,4,5,6,7\}$. The commutator identity (11) implies that $t_{i k}(1) \in\langle x, y\rangle$ for all $i, k, i \neq k$. Hence, $\langle x, y\rangle=$ $\operatorname{SL}(7, \mathbb{Z})$. Now, the fact that $\langle-x, y\rangle=\operatorname{GL}(7, \mathbb{Z})$ follows from Lemma 2.1.

\section{REFERENCES}

[1] L. Di Martino and N. Vavilov, (2,3)-generation of $\operatorname{SL}(n, q)$. I. Cases $n=5,6,7$, Comm. Algebra 22 (1994), no. 4, 1321-1347. MR1261262 (95f:20076)

[2] _ (2,3)-generation of $\mathrm{SL}(n, q)$. II. Cases $n \geq 8$, Comm. Algebra 24 (1996), 487-515. MR $1373489(97 \mathrm{e}: 20067)$

[3] A. J. Hahn and O. T. O'Meara, The classical groups and K-theory, Grundlehren Math. Wiss., Bd. 291, Springer-Verlag, Berlin, 1989. MR1007302 (90i:20002)

[4] M. W. Liebeck and A. Shalev, Classical groups, probabilistic methods, and the (2,3)-generation problem, Ann. of Math. (2) 144 (1996), 77-125. MR.1405944(97e:20106a)

[5] A. Yu. Luzgarëv and I. M. Pevzner, Some facts about the life of GL(5,Z), Zap. Nauchn. Sem. S.Peterburg. Otdel. Mat. Inst. Steklov. (POMI) 305 (2003), 153-162; English transl., J. Math. Sci. (N. Y.) 130 (2005), no. 3, 4729-4733. MR2033620

[6] G. A. Miller, On the groups generated by two operators, Bull. Amer. Math. Soc. 7 (1901), no. 10, 424-426. MR.1557828

[7] Ya. N. Nuzhin, On a question of M. Conder, Mat. Zametki 70 (2001), no. 1, 79-87; English transl., Math. Notes 70 (2001), no. 1-2, 71-78. MR1883773 (2002m:20076)

[8] P. Sanchini and M. C. Tamburini, Constructive $(2,3)$-generation: A permutational approach, Rend. Sem. Mat. Fis. Milano 64 (1994), 141-158 (1996). MR.1397469 (97i:20040)

[9] M. C. Tamburini and J. S. Wilson, On the $(2,3)$-generation of some classical groups. II, J. Algebra 176 (1995), 667-680. MR 1351631 (96h:20085)

[10] M. C. Tamburini, J. S. Wilson, and N. Gavioli, On the (2,3)-generation of some classical groups. I, J. Algebra 168 (1994), 353-370. MR1289105 (95f:20075)

[11] M. C. Tamburini and P. Zucca, On a question of M. Conder, Atti Accad. Naz. Lincei Cl. Sci. Fis. Mat. Natur. Rend. Lincei (9) Mat. Appl. 11 (2000), no. 1, 5-7. MR1797048 (2001i:20097)

[12] M. A. Vsemirnov, Is the group $\mathrm{SL}(6, \mathbb{Z})(2,3)$-generated? Zap. Nauchn. Sem. S.-Peterburg. Otdel. Mat. Inst. Steklov. (POMI) 330 (2006), 101-130; English transl., J. Math. Sci. (N. Y.) 140 (2007), no. 5, 660-675. MR2253569 (2008e:20074)

[13] _ The group GL $(6, \mathbb{Z})$ is (2,3)-generated, J. Group Theory 10 (2007), no. 4, 425-430. MR22334749 (2008f:20118)

St. Petersburg Branch, Steklov Mathematical Institute, Russian Academy of Sciences, Fontanka 27, St. Petersburg 191023, Russia

E-mail address: vsemir@pdmi.ras.ru

Received 10/AUG/2007

Translated by THE AUTHOR 